Quantum Information and Computation, Vol. 0, No. 0 (2003) 000-000

(C) Rinton Press

\title{
ELECTRODE CONFIGURATIONS FOR FAST SEPARATION OF TRAPPED IONS
}

\author{
J. P. HOME \\ Centre for Quantum Computation, Department of Atomic and Laser Physics, Clarendon Laboratory, Parks Road \\ Oxford, OX1 3PU, England \\ A. M. STEANE \\ Centre for Quantum Computation, Department of Atomic and Laser Physics, Clarendon Laboratory, Parks Road \\ Oxford, OX1 3PU, England \\ Received (received date) \\ Revised (revised date)
}

\begin{abstract}
We study the problem of designing electrode structures that allow pairs of ions to be brought together and separated rapidly in an array of linear Paul traps. We show that it is desirable for the electrode structure to produce a d.c. octupole moment with an a.c. radial quadrupole. For the case where electrical breakdown limits the voltages that can be applied, we show that the octupole is more demanding than the quadrupole when the characteristic distance scale of the structure is larger than 1 to 10 microns (for typical materials). We present a variety of approaches and optimizations of structures consisting of one to three layers of electrodes. The three-layer structures allow the fastest operation at given distance $\rho$ from the trap centres to the nearest electrode surface, but when the total thickness $w$ of the structure is constrained, leading to $w<\rho$, then twolayer structures may be preferable.

Keywords: Ion Trap, Multiple Traps

Communicated by: to be filled by the Editorial
\end{abstract}

\section{Introduction}

The application of ion traps to quantum computing [1, 2, 3. has lead to interest in the construction of systems of multiple ion traps situated close together. Experiments have shown that quantum logic gates with good fidelity can be produced between a single pair of ions in a single trap, and such methods can be extended to a few ions in a single trap [4, 5, 6, 7, 8. However, to scale this approach up to the manipulation of many qubits, it is not feasible to try to manipulate large numbers of qubits in a single trap. Instead, it is desirable to have an array of traps, with the possibility to move quantum information between ions in separate traps. A method to move the quantum information which has advantages of relative simplicity and robustness is simply to move the ions themselves [9, 1, 10. To achieve this we need an array of ion traps, with the possibility to move ions between traps. This concept has been demonstrated in an impressive set of experiments [11, 12, 13] which demonstrate its promise. The present work addresses the issue of how to design a basic element of such an array: a region in which a pair of ions can be brought together or separated rapidly, by adjusting d.c. voltages on electrodes. This includes the case of separating into two wells a pair of ions which are initially in a single harmonic well. 
There are further constraints which make this a non-trivial problem:

- The electrode structure should allow a route for ions to be moved into and out of the region of the close pair of trapping centres.

- The surfaces of the electrodes should be kept as far as possible from the trap centres where the ions sit. This is in order both to minimise heating of the motional state of the trapped ions (see below) and to reduce the impact of surface irregularities on the electric potential function experienced by the ions.

- The trap confinement should be tight, i.e. the vibrational frequency $\nu$ of the ions must exceed some desired minimum value. This is in order to maximise the speed at which ions can be moved by displacing the trap centres.

- The electric fields at the electrode surfaces must not exceed the electrical breakdown limitations of the materials.

- The electrode configuration must be capable of being fabricated accurately at the required distance scale. This may require microfabrication techniques which have their own intrinsic limitations.

For diagnostic purposes it may also be useful for the electrode structure to be open in order to allow optical access.

The need to keep the electrode surfaces far away from the ions is largely owing to the observation of an anomalous heating rate in ion traps, which becomes a severe problem with traps of small distance scale. The heating process is not fully understood but appears to be associated with impurities deposited on the electrodes, and studies suggest the heating rate scales as $\rho^{-4}$ where $\rho$ is the distance from trap centre to the nearest electrode surface 14, 11, 15. Electrodes further from the trap centre can also be bigger, which usually implies they are easier to make.

Typical distance scales are 1-10 microns for the separation of the trapped ions, 1-100 microns for the distance to the electrodes [14, 11, 12, 16].

We begin the discussion by considering in section 2 the general problem of a pair of 1 dimensional potential wells which can be moved together or pulled apart while maintaining the tightness, i.e. the normal mode frequencies of a pair of ions trapped in the wells. We argue that the best electrode structure is one which produces an electric octupole potential, which is then 'tweaked' by the addition of quadrupole terms. In section 3 we introduce radial confinement by an a.c. quadrupole, and discuss in general terms the expected scaling of the trap parameters with the size of the electrode structure. We show that for structures whose dimensions are greater than 1 to 10 microns, the most demanding factor in the design is to obtain a large octupole moment, assuming the applied voltages are limited by electrical breakdown of the materials. We then proceed in section 4 to consider the problem of obtaining an electric octupole with a minimum number of electrodes. In section 5 we describe numerical calculations of the electric potential for several types of electrode structure, obtaining the values of two dimensionless factors that give the strength of the three-dimensional d.c. octupole and two-dimensional a.c. quadrupole moment at given breakdown field and trap scale. 
Section [6 then briefly analyses the influence of manufacturing imprecision which breaks the symmetries of an electrode structure and introduces stray electric fields.

Section 7 discusses the main features of the numerical results. For example, we quantify the relative merits of a 3-layer over a 2-layer or single-layer (planar) design. We estimate the impact of requiring the complete structure to be thin (to aid microfabrication). We summarize the optimizations obtained by adjusting the positions and relative sizes of electrodes. Section 8 concludes. The appendices give a discussion of axial micromotion and some general information on charge distributions that produce electric octupoles.

\section{Generic study of $V(x, y, z)$ for two traps close together}

We consider first of all some general properties of the electric potential for two Paul traps located at $(x, y, z)=(0,0, \pm s)$. We assume the vibrational frequencies $\omega_{x}, \omega_{y}, \omega_{z}$ of a single trapped ion are the same for the two traps. The precise relative sizes of $\omega_{x}, \omega_{y}$ and $\omega_{z}$ are not crucial, but it will be useful if $\omega_{x}, \omega_{y} \gg \omega_{z}$. The electrode configurations to be discussed in later sections of the paper will produce the confinement along $z$ primarily by d.c. voltages, and that along $x$ and $y$ primarily by an oscillating (r.f.) quadrupole field. However, we do not need to assume this for the general discussion in this section (for clarification of this point, see the appendix).

Let the distance from the origin to the nearest electrode surface be $\rho$. Since we aim to achieve close traps and far electrodes, we assume $s \ll \rho$. We can then usefully analyse the electric potential $V$ near the origin by a Taylor or a multipole expansion. We further assume that the electrode construction is close to symmetric so that odd order terms in the expansion almost vanish. First let us consider $V$ along the $z$ axis:

$$
V(0,0, z) \simeq V_{0}-E_{0} z+\alpha z^{2}+\beta z^{4}
$$

where we have dropped the cubic term since we assume that it is negligible. The signs of the coefficients $E_{0}, \alpha, \beta$ have been chosen so that $E_{0}$ is the electric field at the origin (assuming $d V / d x=d V / d y=0$ there), and to create a double well potential we need $\alpha<0$ and $\beta>0$ (see figure 1).

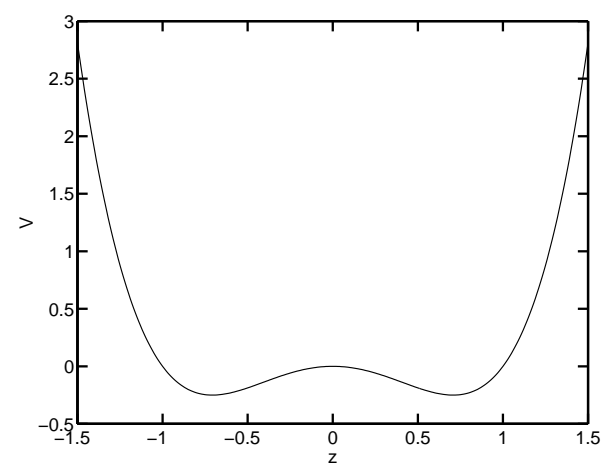

Fig. 1. Plot of $V(z)$ (equation (1) for $V_{0}=0, E_{0}=0, \alpha=-1, \beta=1$.

First suppose there is no linear term, $E_{0}=0$. By solving $\partial V / \partial z=0$ we find for $\alpha<0$ 
the potential wells are centred at

$$
s=\sqrt{\frac{|\alpha|}{2 \beta}} .
$$

Evaluating $\partial^{2} V / \partial z^{2}$ at either well centre gives $\partial^{2} V / \partial z^{2}=4 \alpha$. If the oscillating voltages are so arranged that the $z$ axis is an r.f. null, i.e. $V(0,0, z)$ is constant in time or only has a relatively small oscillation, then we may obtain $\omega_{z}$ for an ion trapped in either well directly from $\partial^{2} V / \partial z^{2}$, giving

$$
-\alpha=\frac{m \omega_{z}^{2}}{4 q}
$$

where $m, q$ are the mass and charge of the ion. For example, at $\omega_{z} / 2 \pi=1 \mathrm{MHz},|\alpha| \simeq 4$ $\mathrm{MV} / \mathrm{m}^{2}$ for the charge to mass ratio of the ${ }^{43} \mathrm{Ca}^{+}$ion. The well depth $V(0)-V(s)=\alpha s^{2} / 2$; e.g. $0.2 \mathrm{mV}(2$ Kelvin) at $s=10 \mu \mathrm{m}$.

In summary, the quadratic term $\alpha$ in the Taylor expansion sets the trap tightness (leading to $\omega_{z}$ ), and the ratio of the quadratic to quartic terms sets the trap separation.

We will arrange for the linear term $E_{0}$ to be small by using electrodes symmetric under reflections in the $x-y$ plane, and then nulling any remaining field if necessary by compensation voltages. Let us calculate next the value of $E_{0}$ which "tips the potential over" just enough so that one of the traps cannot confine ions, i.e. $\partial^{2} V / \partial z^{2}$ goes to zero at a trap centre. This happens when

$$
\left|E_{0}\right| \simeq \frac{4}{3}|\alpha| s .
$$

For example, for ${ }^{43} \mathrm{Ca}^{+}$with $\omega / 2 \pi=1 \mathrm{MHz}$ and $s=10 \mu \mathrm{m},|\alpha| s \simeq 40 \mathrm{~V} / \mathrm{m}$.

\subsection{Cancellation of quadratic term}

Consider the Taylor expansion (1) for a set of electrodes of distance scale $a$. In the absence of special choices or symmetries which make one or more terms disappear, we would obtain

$$
|E| \simeq \frac{\left|V_{0}\right|}{a}, \quad|\alpha| \simeq \frac{\left|V_{0}\right|}{a^{2}},|\beta| \simeq \frac{\left|V_{0}\right|}{a^{4}}
$$

(for example, consider the electric potential due to a point charge, or a line charge, at distance $a$ from the point or line). Therefore, without a special design, the order of magnitude of $s$ is expected to be $s \sim a$. To obtain $s \ll a$ we require $\alpha / \beta \ll a^{2}$, and to obtain this without reducing $\omega_{z}$ we must increase $\beta$. This can be done by constructing an electrode configuration in which the quadratic term $\alpha$ is suppressed compared to $\beta$, and then increasing the voltages on all electrodes together. Assuming the increased voltages are attainable (and we will find they are), the available $\beta$ at given $\alpha$ is limited by electrical breakdown associated with high electric fields at the electrode surfaces.

Assume that the electrodes have reflection symmetry in $x-y, x-z$ and $y$ - $z$ planes, so that the electric field vanishes at the origin. Then odd-order multipole moments of $V(x, y, z)$ vanish, and so do mixed 2nd derivatives such as $\partial^{2} V / \partial x \partial y$ at the origin. Under this assumption, 
we will next show that the required electrode configuration is one which produces, at lowest order in its multipole expansion around the origin, an octupole moment.

An electrode set that can produce $\alpha / \beta \ll a^{2}$ is close to the condition $\alpha \rightarrow 0$. Since we require the possibility to adjust $\alpha$ and $\beta$ (e.g. by adjusting voltages on the electrodes) the same electrode set can also attain $\alpha=0$ (in practice, it may or may not actually be used in that condition). We therefore have to consider electric potential configurations having $\partial^{2} V / \partial z^{2}=0$ and $\partial^{4} V / \partial z^{4} \neq 0$. From Laplace's equation, we then have $\partial^{2} V / \partial y^{2}=-\partial^{2} V / \partial x^{2}$, thus a 2D quadrupole moment in the $x-y$ plane unless these derivatives are also zero. Unless we take special measures to make $\left|\partial^{2} V / \partial x^{2}\right|$ small, we have $\left|\partial^{2} V / \partial x^{2}\right| \sim \beta a^{2} \gg m \omega_{z}^{2} / q$ when $s \ll a$. This implies the field is strongly expelling along some direction in the $x-y$ plane. To permit the ions to remain trapped, either the whole field must be made to oscillate, or an oscillating quadrupole field must be added whose strength is sufficient to overcome this expulsion. If the whole field oscillates then in the pseudo-potential model, the effective values of both $\alpha$ and $\beta$ are reduced, which is counter-productive. If instead a separate oscillating quadrupole is added in the $x-y$ plane (with small effect in the $z$-direction) then its strength must be large, and this leads to electrical breakdown which will constrain the available range of $\beta$.

We conclude that the d.c. electrode structure must be designed to produce small $\left|\partial^{2} V / \partial x^{2}\right|$ (as well as small $\left|\partial^{2} V / \partial z^{2}\right|$ ) and therefore we require an electrode structure at or close to an electric hexapole or octupole configuration. A quantitative statement of this condition is given in sections 3 (eq. (21)) and 3.2 The octupole has the advantage that reflection symmetries which cancel odd-order multipole moments can be used to avoid producing an unwanted electric field (i.e. a contribution to the electric potential varying linearly with distance at the origin), and therefore we will concentrate on that case.

Confinement of the ions in three dimensions is completed by adding to the d.c. octupole field an oscillating 2D quadrupole field whose size is controlled separately. Hence, after reinstating a non-zero value for $\alpha$, we obtain the general form

$$
\begin{aligned}
V(x, y, z, t) \simeq & \alpha\left(z^{2}-\frac{1}{2}\left(x^{2}+y^{2}\right)\right)+\beta V_{4}(x, y, z) \\
& +Q_{\mathrm{ac}} \cos (\Omega t)\left(x^{2}-y^{2}\right)
\end{aligned}
$$

where $\alpha, \beta$ and $Q_{\mathrm{ac}}$ are time-independent, $V_{4}(x, y, z)$ is an octupole potential with $V_{4}(0,0, z)=$ $z^{4}$, and $\Omega$ is the frequency of the a.c. quadrupole providing confinement in the radial direction. Many ion trap configurations in common use are described by ([6), but we have in mind a case where $\beta /|\alpha|$ is as large as possible. We will see later that none of the electrode sets to be discussed realise (6) exactly, because the time-dependent part depends slightly on $z$ as well as $x$ and $y$, and because higher-order terms appear, but it is useful to make clear what we are aiming at.

\subsection{Coulomb repulsion and normal mode frequencies}

If there is one ion in each trap, then the distance between ions is not $2 s$ owing to their mutual Coulomb repulsion. The equilibrium positions are $z= \pm d / 2$ where the separation $d$ is a real positive solution of

$$
\beta d^{5}+2 \alpha d^{3}=\frac{q}{2 \pi \epsilon_{0}}
$$


In order to bring out the general behaviour of solutions to this equation, it useful to express it in the form

$$
\left(\frac{d}{2 s}\right)^{5}+\frac{\alpha}{|\alpha|}\left(\frac{d}{2 s}\right)^{3}=\epsilon
$$

where

$$
\epsilon=\frac{q}{4 \pi \epsilon_{0}|\alpha|(2 s)^{3}}
$$

Therefore the solutions are determined by the sign of $\alpha$ and a single parameter $\epsilon$, which compares the Coulomb repulsion force to the trapping force for ions situated at $\pm s$ in a harmonic well. When the $\beta$ term in (7) dominates, $\epsilon$ is large, and $d \simeq\left(q / 2 \pi \epsilon_{0} \beta\right)^{1 / 5}$. When $\alpha$ is large and positive, i.e. two ions in the same harmonic well, $d \simeq\left(q / 4 \pi \epsilon_{0} \alpha\right)^{1 / 3}$. When $\alpha<0$ and $\epsilon \ll 1$, i.e. a pair of well-separated traps, then $d \simeq s(2+\epsilon)$.

The system of two ions has two normal modes for motion along the $z$ direction: the centre of mass mode of frequency $\omega_{1}$ and the breathing (also called stretch) mode of frequency $\omega_{2}$. These frequencies are given by

$$
\begin{aligned}
& \omega_{1}^{2}=\left(2 \alpha+3 \beta d^{2}\right) q / m, \\
& \omega_{2}^{2}=\omega_{1}^{2}(1+\tilde{\epsilon}),
\end{aligned}
$$

where

$$
\tilde{\epsilon}=\frac{q^{2}}{\pi \epsilon_{0} m \omega_{1}^{2} d^{3}}
$$

( $m$ was defined previously, it is the mass of a single ion).

When the ions are far apart, $\tilde{\epsilon} \rightarrow \epsilon \ll 1$ and the two mode frequencies are both equal to the oscillation frequency of a single ion in either trap. When $\alpha=0, \tilde{\epsilon}=2 / 3$ and when $\alpha \gg 1$ (i.e. a single harmonic well), $\tilde{\epsilon} \rightarrow 2$.

The reason to bring the ions close together is in order to perform a two-ion quantum logic gate in which their Coulomb repulsion provides the interaction energy between the qubits. The gate is fast when the mode frequencies are high and well-separated [17, 18. Therefore we would like a high value of both $\omega_{1}$ and $\tilde{\epsilon}$. Suppose the ions are initially in traps far apart, so that $\tilde{\epsilon} \ll 1$. Then the vibrational frequencies are almost equal and are given by the value of $\alpha$ through eq. (3). To bring the ions together, the voltages on the electrodes may be changed so that $\beta$ gets larger while $\alpha$ stays the same size. Eventually electrical breakdown prevents further increase of $\beta$. If at this point the values of $\omega_{1}$ and $\tilde{\epsilon}$ are high enough to give an acceptable gate speed, and if it is possible to apply the logic-gate laser pulses to ions in the separation zone, then the gate is performed and the ions are subsequently separated. Otherwise, to bring the ions into a common trap, $|\alpha|$ must be lowered and one accepts an unavoidable reduction in mode frequencies while the potential barrier between the twin traps is lowered, see figure 2 After $\alpha$ passes through zero and becomes positive, the mode frequencies increase again and $\tilde{\epsilon}$ rises towards its maximum value of 2 . The ions can then be moved as a pair to a convenient (e.g. less noisy) region of the complete array, for the logic-gate laser pulses to be applied. 


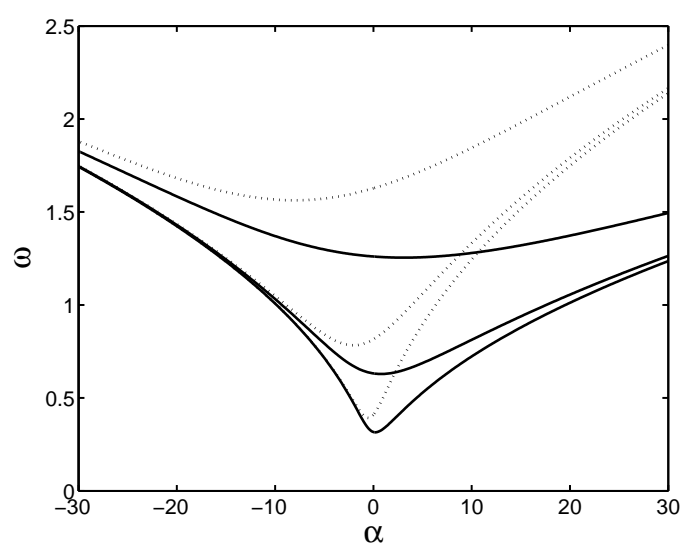

Fig. 2. Plot of the centre-of-mass frequency $\omega_{1}$ (solid curve) and stretch mode frequency $\omega_{2}$ (dotted curve) vs. $\alpha$ for three different values of $\beta$. The curves shown are for $\beta=100,10,1$ in arbitrary units, the higher value of $\beta$ giving the higher normal mode frequencies. The figure shows the generic behaviour, the units are arbitrary.

At the condition $\alpha=0$, the full analysis is tractable and one finds

$$
\omega_{1}=\sqrt{\frac{3 q}{m}}\left(\frac{q}{2 \pi \epsilon_{0}}\right)^{1 / 5} \beta^{3 / 10}
$$

and $\tilde{\epsilon}=2 / 3$ as mentioned above.

In the rest of this paper we will consider methods to produce this condition, i.e. an octupole potential, with an added oscillating quadrupole potential in the $x-y$ plane, as in (6). In practice the electrode structures to be considered depart from (6) primarily in one respect, namely that the oscillating term includes a $z$-dependence, i.e.

$$
\begin{aligned}
V(x, y, z, t) \simeq \alpha & \left(z^{2}-\frac{1}{2}\left(x^{2}+y^{2}\right)\right)+\beta V_{4}(x, y, z) \\
& +\cos (\Omega t)\left(\alpha_{x} x^{2}-\alpha_{y} y^{2}+\alpha_{z} z^{2}\right)
\end{aligned}
$$

where we introduce the parameters $\alpha_{x}, \alpha_{y}, \alpha_{z}$ to characterise the 3 -dimensional a.c. quadrupole. If $\alpha_{x} \neq \alpha_{y}$ then necessarily $\alpha_{z} \neq 0$, and in this case there is axial micromotion. It is shown in the appendix that this leads to a Mathieu equation, which can be solved in the standard way as a combination of slow secular motion and fast micromotion 20, 21]. The secular motion can be modelled as motion in a pseudo-potential which takes into account the influence of both the d.c. and the a.c. (ponderomotive) terms. The above analysis then remains correct (within the pseudopotential approximation) as long as we replace $\alpha$ by $\alpha^{\prime}$, where $\alpha^{\prime}$ is the coefficient of the $z^{2}$ term in the total effective potential. For the structures discussed in this paper, it is found that the electrode voltages required to produce $\alpha^{\prime}=0$ are very close to those required for $\alpha=0$, hence changing from one condition to the other has little effect on $\beta$. At $\alpha^{\prime}=0$ the secular frequency is completely determined by the value of $\beta$ through (13).

\section{Radial r.f. confinement and scaling}

Consider the potential (6). A pair of trapped ions is confined in the radial ( $x$ and $y$ ) 
direction by the oscillating quadrupole field. To first approximation we may ignore the effect of the octupole potential on the radial motion, then this motion is described by a Mathieu equation. In the pseudo-potential model, for $\alpha=0$, the secular frequency of the centre-of-mass vibrational motion in the radial direction is given by [19, 20, 21]

$$
\omega_{r}=q_{r} \Omega / 2 \sqrt{2},
$$

where

$$
q_{r}=\frac{4 q Q_{\mathrm{ac}}}{\Omega^{2} m}
$$

is the Mathieu $q$-parameter. For stable motion, $q_{r}$ must not be large; values in the range 0.1 to 0.5 are typical. This constrains the applied r.f. frequency $\Omega$ and hence we obtain

$$
\omega_{r}=\left(\frac{q_{r} q}{2 m} Q_{\mathrm{ac}}\right)^{1 / 2}
$$

At the octupole condition, equations (13) and (17) give the centre of mass vibrational frequencies for motion in the axial and radial directions respectively. Since we want the ions to align themselves along the $z$ axis, we require $\omega_{r}>\omega_{1}$ (and $\omega_{r} \gg \omega_{1}$ is desirable). This sets a lower limit on the required value of $Q_{\mathrm{ac}}$ for given $\beta$ :

$$
Q_{\mathrm{ac}}=\left(\frac{\omega_{r}}{\omega_{1}}\right)^{2} \frac{6}{q_{r}}\left(\frac{q}{2 \pi \epsilon_{0}}\right)^{2 / 5} \beta^{3 / 5} .
$$

It is useful to examine the way these parameters scale with $\rho$, the distance from the origin to the nearest electrode surface, and $E_{\max }$, the largest electric field at any electrode surface. For a given electrode geometry $\rho$ sets the distance scale of the electrodes. To keep $E_{\max }$ as small as possible, the electrode surfaces should have as large a radius of curvature as possible, and therefore their radii should be allowed to increase with $\rho$. In other words the complete structure of the electrodes is assumed to scale with $\rho$. Nevertheless for a given electrode set at a given value of $E_{\max }, \beta$ and $Q_{\mathrm{ac}}$ still have a range of possible values, because it is possible to choose a range of values for the ratio between the sizes of the oscillating and the d.c. voltages. Typically the variation is such that there is a competition between high $\beta$ and high $Q_{\mathrm{ac}}$.

In order to obtain the main features by a relatively simple analysis, we define two geometric factors $\gamma$ and $\mu$ such that

$$
\begin{aligned}
& \text { for } Q_{\mathrm{ac}}=0, \quad \beta=\frac{\gamma E_{\mathrm{max}}}{\rho^{3}}, \\
& \text { for } \beta=0, \quad Q_{\mathrm{ac}}=\frac{\mu E_{\max }}{\rho} .
\end{aligned}
$$

Thus $\gamma$ and $\mu$ tell us about the maximum values of $\beta$ and $Q_{\mathrm{ac}}$ available for a given electrode geometry, but we keep in mind that it is not possible to have both these maximum values at once.

We can now quantify the desirability of the octupole potential, as compared with other possibilities. If the electrode structure is not designed to produce small or zero d.c. quadrupole, then it will produce a d.c. quadrupole potential in the $x y$ plane of strength $\alpha_{\mathrm{r}}^{\mathrm{dc}} \simeq \rho^{2} \beta$. To 
achieve radial confinement, we require $\omega_{r}^{2}$ as given by (17) to exceed $2 \alpha_{\mathrm{r}}^{\mathrm{dc}} q / m$. Hence the condition for stable radial confinement is $Q_{\mathrm{ac}}>4 \rho^{2} \beta / q_{r}$ and therefore

$$
\mu>\frac{4}{q_{r}} \gamma
$$

It is interesting that this condition is scale-independent. Since it is satisfied for large $\mu$ or small $\gamma$, it shows that suppressing the d.c. quadrupole in all directions is not necessary for electrode structures which naturally produce a strong radial confinement or a weak octupole. However, we are interested in making both parameters large, and it will be shown that the structures offering high values of both parameters do not satisfy (21), therefore the suppression of the d.c. quadrupole is useful.

Suppose the ions each have one electronic unit of charge and let $A$ be the mass number, then for a trap at the octupole condition $(\alpha=0)$, eq. (13) gives

$$
\frac{\omega_{1}}{2 \pi}[\mathrm{MHz}] \simeq \frac{840}{\sqrt{A}} \frac{\left(\gamma E_{\max }\right)^{3 / 10}}{\rho^{9 / 10}} .
$$

where $E_{\max }$ is in $\mathrm{V} / \mu \mathrm{m}$ and $\rho$ is in $\mu \mathrm{m}$. Note that this mode frequency scales almost linearly with $1 / \rho$, and is relatively insensitive to $\gamma$ and $E_{\max }$. The radial frequency (eq. (17)), on the other hand, scales as

$$
\omega_{r} \propto \frac{\mu^{1 / 2}}{\rho^{1 / 2}}
$$

This difference in the scaling of frequency with trap size, for the radial and axial motion, is illustrated in figure 3 The figure shows the frequencies $\omega_{1}$ (eq. (22)) and $\omega_{r}$ (eq. (17)) plotted as a function of $\rho$ for two example electrode configurations discussed in section 5 In order that a single graph can show the behaviour for all ion species, the vibrational frequencies are shown in units of a basic frequency $\omega_{0}$ which depends on the charge/mass ratio of the ion. We define $\omega_{0}$ to be the radial secular frequency (eq. (17)) for an ion in a trap having $Q_{\mathrm{ac}}=10^{8}$ $\mathrm{Vm}^{-2}$ and $q_{r}=0.3 . \omega_{0} / 2 \pi$ takes the value $923 \mathrm{kHz}$ for ${ }^{43} \mathrm{Ca}^{+}, 574 \mathrm{kHz}$ for ${ }^{111} \mathrm{Cd}^{+}$and 2017 $\mathrm{kHz}$ for ${ }^{9} \mathrm{Be}^{+}$.

Each point where the lines cross on figure 3 identifies a distance scale $\rho_{c}$. It is found by substituting (19) and (20) into (18) and solving for $\rho$ :

$$
\rho_{c} \equiv \frac{\gamma}{\mu}\left(\frac{1}{\gamma \mu}\right)^{1 / 4}\left(\frac{1}{6 q_{r}}\right)^{5 / 4}\left(\frac{\omega_{r}}{\omega_{1}}\right)^{5 / 2} L_{0}
$$

where

$$
L_{0}=36\left(3 q / \pi \epsilon_{0} E_{\max }\right)^{1 / 2} .
$$

The distance scale $\rho_{c}$ answers the question, "which motional frequency, the radial or the axial, will be the more challenging to achieve when we design the electrode structure?" It thus helps to establish priorities in the task. Below $\rho_{c}$ the challenge is to increase the radial frequency, while above $\rho_{c}$ the challenge is to increase the axial frequency. This is because in order to maintain a linear ion configuration, below $\rho_{c}$ the trap must be weakened in the axial direction, 

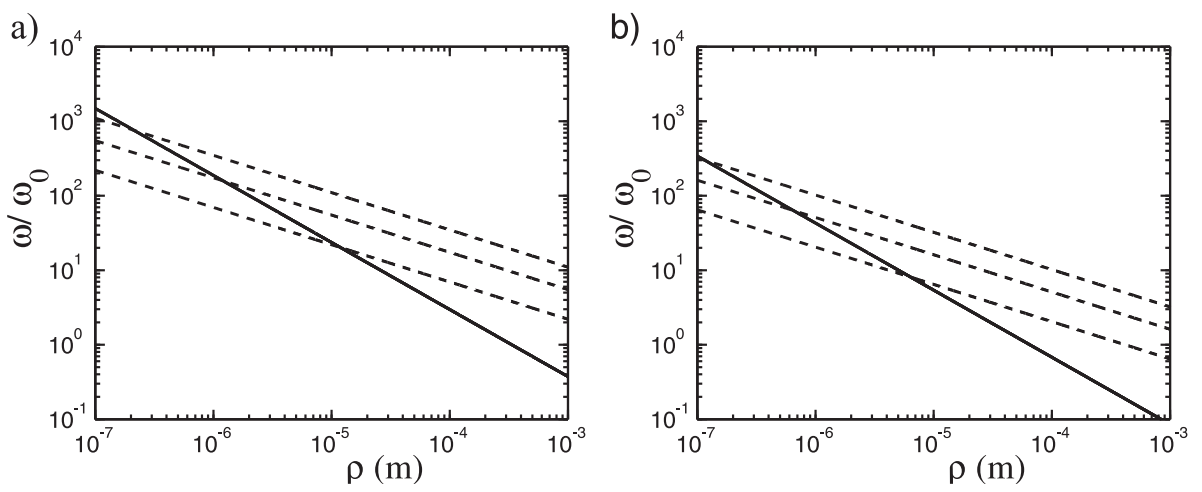

Fig. 3. Centre-of-mass vibrational frequencies for axial motion of two ions in a d.c. octupole potential (solid line, $\omega_{1}$ ) and radial motion of two ions in a linear Paul trap (upper dashed line, $\left.\omega_{r}\right)$. The frequency unit $\omega_{0}$ is defined in the text. (a): electrode arrangement 2.1 , (b): electrode arrangement 5.1. The frequencies are obtained using values of $\gamma$ and $\mu$ presented in section 5 and using $E_{\max }=10^{8} \mathrm{~V} / \mathrm{m}$. The lower two dashed lines are $\omega_{r} / 2$ and $\omega_{r} / 5$; these permit the conditions $\omega_{1}=\omega_{r} / 2$ and $\omega_{1}=\omega_{r} / 5$ to be identified easily.

by operating it with $\beta$ below the maximum available value, and therefore in this regime $\mu$ gives the limit on the performance.

We now show that the value of $\rho_{c}$ is of order microns in practice.

In the expression (24) the factor $\left(1 / 6 q_{r}\right)^{5 / 4} \simeq 0.5$ to 2 for a choice of Mathieu $q_{r}$ parameter in the range 0.1 to 0.3 . The combination of geometric factors in (24) has a value in the range 0.1 to 2 for the structures described below (with the exception of those shown in figures 4 and 5 which we reject as unsatisfactory owing to their poor radial confinement). Therefore we find $\rho_{c} \simeq(0.3$ to 22$) L_{0}$ for $\omega_{r}=2 \omega_{1}$. In practice one would choose $\omega_{r} / \omega_{1}$ in the range 2 to 5 in order to keep the ions near the axis without restricting $\omega_{1}$ too much. Increasing $\omega_{r} / \omega_{1}$ from 2 to 5 creates an order of magnitude increase in $\rho_{c}$.

In practice we are concerned with singly charged ions, and therefore $L_{0}$ is a distance scale set purely by the maximum electric field allowed in the structure. For example when $E_{\max }=$ $10^{9} \mathrm{~V} / \mathrm{m}$ (the order of magnitude when field emission may occur [22, 23]), $L_{0} \simeq 0.15 \mu \mathrm{m}$. This field depends mainly on the materials, and partly on the geometry (for example a larger field may be allowed across a vacuum gap than across an insulating material surface). However, owing to the square root in (25) the dependence is not strong.

Taking $q_{r}=0.3$ and combining these observations, it is seen that for a variety of structures, $\rho_{c}<10 \mu \mathrm{m}$ for $\omega_{r}=5 \omega_{1}$ at high electric field, and $\rho_{c}<2 \mu \mathrm{m}$ for $\omega_{r}=2 \omega_{1}$ and more modest fields. This implies that for ion traps designed for quantum computing experiments, the more demanding problem is currently to obtain a large d.c. octupole moment, but the radial confinement may become the more demanding problem in the future as distance scales are reduced. The values of $\rho$ where the lines cross in figure 3 illustrate these points.

Another important distance related to $L_{0}$ is the distance between the ions when the electrodes are close to the octupole configuration. Using (17) and (19) we obtain

$$
d=\frac{\rho}{6}\left(\frac{L_{0}^{2}}{\gamma \rho^{2}}\right)^{1 / 5} .
$$


Therefore $d \ll \rho$ when $\rho \gg L_{0} / \gamma^{1 / 2}$, which is usually the case. For example, putting $\gamma=0.02$ (c.f. table 2 below) and $L_{0}=0.2 \mu \mathrm{m}, d \ll \rho$ when $\rho \gg 16 \mathrm{~nm}$. For electrodes of micron scale or above, therefore, the available confinement is such that when the electric potential is just at the point of producing a potential hill between the ions, the distance between them is much smaller than the distance to the electrodes. This implies that the Taylor expansion we have adopted is valid.

\subsection{Scaling of heating effects}

The main reason why one would not make a quantum computer faster simply by fabricating the traps at a smaller distance scale is the fact that heating problems will be exacerbated. Heating rates observed in various studies scaled as $\rho^{-4}$ [16, 14] and the scaling expected for heating caused by Johnson noise is $\rho^{-2}$. We will treat some general scaling law $\rho^{-k}$, and we assume this heating depends on the electrode structure only through the distance scale $\rho$. The time to separate a pair of ions scales as $1 / \omega_{1}$, therefore the number of phonons' worth of heating per split time, $\Delta n$, is proportional to $1 /\left(\omega_{1} \rho^{k}\right)$. This gives a good guide to the amount of cooling which will be needed to obtain a steady average temperature in the computer. Using equation (22) we obtain

$$
\Delta n \propto \frac{\rho^{-k+9 / 10}}{\gamma^{3 / 10}}
$$

For a given electrode geometry, $\gamma$ is fixed, hence this scaling law governs the allowed distance scale $\rho$. If we take $\Delta n$ as a fixed quantity, then $\rho \propto \gamma^{3 /(9-10 k)}$. Substituting this behaviour into equation (22) we find the following scaling for the trap frequency in terms of $\gamma$ :

$$
\omega_{1} \propto \gamma^{3 k /(10 k-9)}
$$

For example, for $k=2$ the scaling is $\omega_{1} \propto \gamma^{0.545}$, for $k=4$ the scaling is $\omega_{1} \propto \gamma^{0.387}$. When the radial rather than the axial frequency is the harder to achieve, then a similar analysis may be used to relate $\omega_{r}$ to $\mu$.

\subsection{Study of a non-octupole trap structure in current use}

An example of an ion trap in current use which has been used to separate ions is that operated at NIST Boulder for teleportation and other experiments 12. It is a two-layer trap with 5 d.c. electrodes in two opposing quadrants, and the r.f. electrodes in the other quadrants. With $z$ along the trap axis, the ends of the electrodes lie at $x=a, y=a$ where $a=100 \mu \mathrm{m}$. It is not possible to produce an octupole using such an electrode configuration (this requires 7 d.c. electrodes, see section [5.5].

We studied this structure by modelling it and solving Laplace's equation numerically. The calculations were performed using Simion [24] and CPO [25]. Simion uses a finite difference method to find the potential of a regular 3D array of points. The array is iteratively relaxed so as to satisfy Laplace's equation and the boundary conditions, until the fractional changes per iteration are all below $5 \times 10^{-3}$. However, from simulations of analytically solvable cases, we found that the error in electric fields close to spherical and cylindrical electrodes could be as high as $10 \%$. CPO uses a surface charge method, and we found it to be considerably faster and also much more precise. All the numerical results presented in this paper were obtained using CPO. 
To calculate a value for the parameter $\gamma=\rho^{3} \beta / E_{\max }$, we numerically solve Laplace's equation for the given electrode set-up, and then extract $\beta=(1 / 24) \partial^{4} V / \partial z^{4}$ evaluated at the origin, and $E_{\max }$ by examining the gradient of $V$ near the surfaces of all the electrodes.

In order to find $\mu$, calculations were performed with the r.f. electrodes at a voltage of $10^{6} \mathrm{~V}$ which is large compared to the d.c. voltages of $\sim 1 \mathrm{~V}$ used for the d.c. electrodes. The value of the radial confinement $\alpha_{r} \equiv Q_{a c}$ was extracted, along with the value of the maximum field $E_{\max }^{\mu}$ at the surface of the electrodes. The radial geometrical parameter $\mu$ was then calculated using equation (20).

For the model of the NIST trap, first the r.f. electrodes were grounded and the voltages on the d.c. electrodes were adjusted to satisfy $\partial^{2} V / \partial x \partial y=\partial^{2} V / \partial y \partial x=\partial^{2} V / \partial z^{2}=0$. We then found that $\partial^{2} V / \partial y^{2}=-\partial^{2} V / \partial x^{2}=0.02 \mathrm{Va}^{-2}, \beta=0.01 \mathrm{Va}^{-4}$ and $E_{\max }=11.1 \mathrm{Va}^{-1}$. The NIST trap has $\rho=1.38 a$, hence we deduce $\gamma=0.0024$. The same procedure was carried out with $\partial^{2} V / \partial x^{2}=\partial^{2} V / \partial y^{2}=\partial^{2} V / \partial z^{2}=0$ but the remaining (undesirable) quadrupole terms were found to be 10 times larger. In each case we then raised the r.f. electrodes to a high voltage in order to find $\mu$, obtaining the value 0.11 in both cases.

We observe that the d.c. quadrupole which exists in the $x-y$ plane when $\partial^{2} V / \partial z^{2}=0$ in this trap has a strength very close to $a^{2} \beta$, i.e. it is just as large as we would expect it to be from general scaling considerations when the structure is not designed to eliminate it. The values of $\gamma$ and $\mu$ satisfy condition (21) for $q_{r}>0.006$, therefore in this trap the radial confinement from the a.c. potential can overcome the d.c. anticonfinement before the r.f. voltage is restricted by electrical breakdown. This can be regarded as owing to a low value for $\gamma$. However, the presence of the d.c. quadrupole remains unwelcome, because it results in a large increase in the required r.f. voltages.

To estimate the maximum axial and radial frequencies which could be achieved at the point of ion separation, we use $q_{r}=0.3$ and assume that the maximum field allowed at the surface of the electrodes is $10^{8} \mathrm{Vm}^{-1}$. Then the voltages on the d.c. electrodes are 67 , $153,509 \mathrm{~V}$ on the central, intermediate and outer electrodes respectively. For $\omega_{r} \geq 3 \omega_{z}$ the r.f. voltage amplitude is $V_{r f}=557 \mathrm{~V}$. In the case of beryllium ions, these conditions lead to motional frequencies $\left(\omega_{x}, \omega_{y}, \omega_{z}\right)=2 \pi \times(7,30,2) \mathrm{MHz}$. The large difference between $\omega_{x}$ and $\omega_{y}$ shows that most of the voltage on the r.f. electrodes is being used to overcome the d.c. anticonfinement along $x$. If the d.c. quadrupole were eliminated, then one would obtain $\omega_{x} / 2 \pi, \omega_{y} / 2 \pi$ both equal to $20 \mathrm{MHz}$. In that case one would have the option of reducing the r.f. voltage by an order of magnitude.

\section{Realising octupoles}

In this section we discuss general methods to obtain an octupole moment at the origin of coordinates. This acts as a guide in the design of the electrodes.

Three examples of octupole potentials are the axially symmetric octupole:

$$
V=z^{4}-3 z^{2}\left(x^{2}+y^{2}\right)+\frac{3}{8}\left(x^{2}+y^{2}\right)^{2}
$$

an octupole with cubic symmetry:

$$
V=x^{4}+y^{4}+z^{4}-3\left(x^{2} y^{2}+x^{2} z^{2}+y^{2} z^{2}\right)
$$


and the 2-dimensional octupole in the $x$ - $z$ plane:

$$
V=z^{4}+x^{4}-6 z^{2} x^{2} .
$$

The axially symmetric octupole can be produced by a set of 2 (+ve) end-cap and 3 ring $(2$ -ve, central one + ve) electrodes, all shaped to follow contours of $V$. The 2D octupole can be produced by a set of $8(4+\mathrm{ve}, 4$-ve) rod electrodes parallel to the $y$ axis and located at the corners of an octagon. These two examples of pure octupoles give a useful pointer to the required distribution of image charge.

The methods and image charge distributions listed below do not produce pure octupoles, but have an octupole as the leading term in the multipole expansion of their potential near the origin.

\subsection{Use of symmetry}

To produce an octupole at a point $\underline{r}, 19$ constraints have to be satisfied. These are that the first, second and third derivatives of the potential at this point in three orthogonal directions should vanish. Many of these can be satisfied by the introduction of symmetry to the charge distribution producing the potential. This can take the form of either rotation symmetry about an axis passing through $\underline{r}$, or reflection symmetry in a plane containing the point $\underline{r}$.

A single reflection in a plane containing the point $\underline{r}$ produces zero odd-order moments in a direction perpendicular to the plane, and also forces the mixed second derivatives such as $\partial^{2} V / \partial x_{i} \partial x_{j}$ to vanish, where $x_{i}$ lies within and $x_{j}$ lies perpendicular to the plane of reflection. This leaves 12 constraints to be satisfied.

Two reflections in orthogonal planes containing $\underline{r}$ produces zero odd-order moments in the directions normal to the two planes, and all mixed second derivatives vanish. This leaves 7 constraints to be satisfied.

Two-fold rotation symmetry about an axis passing through $\underline{r}$ causes the odd-order derivatives of the potential in all directions perpendicular to the axis to vanish, reducing the number of constraints to 9 .

Four-fold rotation symmetry about an axis passing through $\underline{r}$ makes the second derivatives in directions perpendicular to the axis equal, hence the number of constraints is reduced to 5 .

Combinations of the above can be used to reduce the number of constraints further. Reflection symmetry in three orthogonal planes all containing $\underline{r}$ reduces the constraints to 3 , on the non-mixed second derivatives $\partial^{2} V / \partial x_{i}^{2}$. Combining a two-fold rotation about an axis passing through $\underline{r}$ with a reflection in a plane normal to the axis and containing $\underline{r}$ reduces the constraints to 4 (the non-mixed second derivatives and the derivative $\partial^{2} V / \partial x_{i} \partial x_{j}$, where $x_{i}$ and $x_{j}$ are orthogonal directions perpendicular to the rotation axis). A four-fold rotation symmetry about an axis passing through $\underline{r}$ combined with a reflection in a plane normal to the axis and containing $\underline{r}$ leaves 2 constraints (on the three non-mixed second derivatives, two of which are equal).

Finally, Laplace's equation reduces the degrees of freedom of the non-mixed second derivatives $\partial^{2} V / \partial x_{i}^{2}$ by one.

\subsection{Image charge constructions}

(A). An octupole with cubic symmetry is produced by any arrangement of charge having 
cubic symmetry. Proof: the symmetries under reflections in three orthogonal planes imply the odd-order multipole moments vanish, and so do mixed second derivatives of $V$ such as $\partial^{2} V / \partial x \partial y$; the symmetries under rotation through $90^{\circ}$ imply the coefficients of $x^{2}, y^{2}$ and $z^{2}$ in a Taylor expansion of the potential at the centre of symmetry are all the same, and therefore by Laplace's equation they all vanish.

(B). An octupole with cylindrical symmetry is produced by any arrangement of charge having cylindrical symmetry, reflection symmetry in $z=0$, and placed such that $\partial^{2} V / \partial z^{2}=0$ (where $z$ is the symmetry axis). Proof: the second derivatives w.r.t. $x$ and $y$ must be equal by symmetry and therefore by Laplace's equation they also vanish.

(C). An octupole is produced by any arrangement of charge having four-fold rotational symmetry in the $x-z$ plane, reflection symmetry in $y=0$, and arranged so that $\partial^{2} V / \partial y^{2}=0$. The reasoning is the same as in the previous case. The resulting octupole potential is a combination of the axially symmetric case (29) and the 2-dimensional case (31) oriented such that $y$ is the common axis.

(D). An octupole can be produced, starting with any arrangement of charge $\rho(x, y, z)$, by the following recipe. First ensure reflection symmetries in order to cancel odd multipole moments, by forming

$$
\bar{\rho} \equiv \sum_{i=0}^{1} \sum_{j=0}^{1} \sum_{k=0}^{1} \rho\left((-1)^{i} x,(-1)^{j} y,(-1)^{k} z\right) .
$$

Next, form

$$
\bar{\rho}^{\prime} \equiv \bar{\rho}(x, y, z)-\bar{\rho}(x / f, y / f, z / f) .
$$

where $f \neq 1$ is a numerical factor. $\bar{\rho}^{\prime}$ produces an octupole at the centre of symmetry. This can be proved by evaluation of the second derivatives of $V(\mathbf{r}) \propto \int \bar{\rho}^{\prime}\left(\mathbf{r}^{\prime}\right) /\left|\mathbf{r}-\mathbf{r}^{\prime}\right| d \tau$ at the origin. A proof giving further physical insight is as follows. $\bar{\rho}(x / f, y / f, z / f)$ produces the same functional form of $V(x, y, z)$ as $\bar{\rho}(x, y, z)$ but on a distance scale expanded by a factor $f$. Modelling $\bar{\rho}$ as a set of point charges, each charge in the rescaled distribution is now further from the origin by a factor $f$, and is increased in size by a factor $f^{3}$. Since the second derivatives of $V$ due to a point charge go as (distance) $)^{-3}$, it follows that the second derivatives of the new potential are equal and opposite to those of the original one, QED. The fourth derivatives scale as (distance) $)^{-5}$, so

$$
\frac{\partial^{4} \bar{V}^{\prime}}{\partial x_{i}^{4}}=\left(1-\frac{1}{f^{2}}\right) \frac{\partial^{4} \bar{V}}{\partial x_{i}^{4}}
$$

where $\bar{V}^{\prime}(\bar{V})$ is the potential due to $\bar{\rho}^{\prime}(\bar{\rho})$ respectively.

A natural extension of this method can produce higher order multipole configurations.

(E). An octupole can be formed by taking almost any charge distribution $\rho(x, y, z)$, and displacing and reflecting it in three dimensions. A general displacement to $\rho\left(x-x_{0}, y-y_{0}, z-\right.$ $z_{0}$ ) has three free parameters $\left(x_{0}, y_{0}, z_{0}\right)$. One can be absorbed into an overall scale factor, leaving two. This is just enough free parameters to allow the two constraints

$$
\begin{aligned}
& \frac{\partial^{2} V}{\partial x^{2}}(0,0,0)=0 \\
& \frac{\partial^{2} V}{\partial y^{2}}(0,0,0)=0
\end{aligned}
$$


to be satisfied, where $V$ is the potential due to $\rho\left(x-x_{0}, y-y_{0}, z-z_{0}\right)$, except in rare cases when the form of $\rho$ leads to no solution. The nulling of $\partial^{2} V / \partial z^{2}$ follows from Laplace's equation. Finally, introduce reflection symmetry as in (32), which causes odd-order moments and mixed second derivatives $\left(\partial^{2} V / \partial x \partial y\right.$ etc.) to vanish.

Another way to understand this construction is to argue that most charge distributions produce a potential with zero second derivatives with respect to fixed orthogonal axes at some point in space; it suffices to place the origin at such a point and then introduce reflection symmetry. For example, if $\rho$ corresponds to a single point charge, this construction leads to point charges on the corners of a cube. More generally if $\rho(x, y, z)$ lies in the $x$ - $z$ plane, this construction leads to a distribution of charge in two parallel planes at $y= \pm y_{0}$.

(F). All the above constructions involve constraints on the locations of the image charges in addition to the basic assumption of three-fold reflection symmetry. Our next construction does not. An octupole can always be formed (with rare exceptions arising in pathalogical cases) by a charge distribution $\bar{\rho}$ with reflection symmetry as in (32), based on

$$
\rho(x, y, z)=\rho_{0}+f_{1} \rho_{1}+f_{2} \rho_{2}
$$

where $\rho_{i}(x, y, z)(i=0,1,2)$ are three different charge distributions located anywhere in the positive octant, and $f_{1}$ and $f_{2}$ are parameters whose values are given by solving the simultaneous equations

$$
\left.\begin{array}{l}
\frac{\partial^{2} V_{0}}{\partial x^{2}}+f_{1} \frac{\partial^{2} V_{1}}{\partial x^{2}}+f_{2} \frac{\partial^{2} V_{2}}{\partial x^{2}}=0 \\
\frac{\partial^{2} V_{0}}{\partial z^{2}}+f_{1} \frac{\partial^{2} V_{1}}{\partial z^{2}}+f_{2} \frac{\partial^{2} V_{2}}{\partial z^{2}}=0
\end{array}\right\}
$$

(evaluated at the origin), in which $V_{i}$ are the potentials due to $\rho_{i}$. This construction uses the same property invoked in the previous one, namely that after cancellation of odd-order moments there are only two constraints (35) we need to satisfy. This implies that we only need two free parameters, and these are provided by $f_{1}$ and $f_{2}$.

$(\mathrm{G})$. When we abandon a reflection symmetry, the octupole can be regained by inserting further charges. For example, suppose we only have reflection symmetry in the $x-y$ and $y-z$ planes. This is the case, for example, when all the charges are located in or on a single substrate, with the octupole centred above the top surface of the substrate. Then in addition to (35) we have 4 further constraints, on $\partial V / \partial y, \partial^{3} V / \partial y^{3}, \partial^{3} V / \partial x^{2} \partial y, \partial^{3} V / \partial z^{2} \partial y$. One or more of these can be satisfied by a careful placement of a single charge distribution. Alternatively, charges are placed at any convenient position, and then their magnitudes are adjusted, similar to construction (F). To obtain the desired behaviour of the trapping centres, we don't need to insist on an octupole, however. Of these further constraints, only $\partial V / \partial y=0$ is strictly necessary.

\subsection{Application to electrode design}

The distributions of charge discussed above are to be realised on the surfaces of a set of conducting electrodes at fixed voltages (or else they are image charge distributions which lead to the same equipotential surfaces). We wish to avoid complicated electrode shapes so we restrict ourselves to electrodes approximating to simple combinations of lines, rings, or sheets.

In order to satisfy $N$ constraints on derivatives of $V(x, y, z)$ merely by adjusting voltages on electrodes, it is necessary to have $N+2$ electrodes, since one defines a voltage zero, and 
the next one sets an overall scale factor. In order to produce radial confinement, a set of r.f. electrodes is introduced around (or in between) the d.c. ones which create the octupole. These r.f. electrodes also serve to define the d.c. zero. Thus, in construction (F) we require one r.f. voltage at d.c. ground, and 3 adjustable d.c. voltages; in construction $(\mathrm{G})$ we require one r.f. voltage at d.c. ground, and 7 adjustable d.c. voltages.

Most of the constructions presented in the previous section can be ruled out, as follows.

The case of cubic symmetry (A) involves 8 electrodes, but to preserve precise symmetry these would have to lie along the edges or the extended major diagonals of a cube, which is difficult to fabricate, and a linear r.f. electrode set would also break the symmetry.

Case (B) can be realised with just two ring-shaped electrodes. This involves the fewest electrodes. For a Penning trap one would choose the magnetic field direction as the axis of symmetry of the rings. For a Paul trap the need to provide an additional r.f. quadrupole producing radial confinement in the $x-y$ plane suggests that there will be further electrodes parallel to the $z$ axis. This implies the fabrication is more straightforward if the rings are placed around another axis, which we take to be the $y$ axis, so that all the electrodes lie in a set of parallel planes. The r.f. electrodes then destroy the circular symmetry. To regain a simple design, the rings can be replaced with rectangular loops, c.f. construction (C).

The other cases can all be fabricated in layers. The method of (33) (case (D)) involves up to 16 electrodes in total or 8 if they all lie in a plane; the method by displacement (case (E)) involves 8 electrodes in two parallel planes, the method of (36) (case (F)) involves up to 24 electrodes in general, or up to 12 if they all lie in a plane, which we take to be the $x-z$ plane. This can be reduced to 10 if one pair lies on the $x$ axis. We avoid placing electrodes along the $z$ axis, because we want to be able to bring ions into and out of the structure by moving them along this axis.

The minimal number of electrodes for a perfectly constructed system is not the only consideration, however. Manufacturing imprecision, patch potentials, and the desire to adjust the trap separation all imply that further electrodes will be needed to trim the system. The electrode structures which rely on precise construction to produce an octupole (cases (A)-(E)) require more of these extra electrodes than those which depend on variable voltages (cases $(\mathrm{F})$ and $(\mathrm{G})$ ).

Screening effects arise when we consider conducting electrodes rather than merely charge distributions. The innermost electrodes tend to act like a Faraday cage to modify and screen the effects of the outer ones. Let $P$ be the centre of the region in which we require a certain potential form (here, $P$ is at the origin). A rough rule of thumb is that if there is not an unobscured line of sight from $P$ to a given electrode, then the influence of that electrode on the potential at or near $P$ is greatly modified by the obstructing electrode. However, the outer charge distribution in (33) is, by construction, exactly on an extended line from the origin to the inner charge distribution. Therefore an electrode set built with the aim of realising the recipe (D) suffers from screening effects and it is difficult to obtain a surface charge distribution of the form (33).

In the remainder of this paper we will concentrate on the case $(\mathrm{F})$ of 10 electrodes all in the $x-z$ plane, of which one pair lies along the $x$ axis, the case (E) of 8 or more electrodes in two planes at $y= \pm y_{0}$, and the case $(\mathrm{G})$ of an octupole situated above all the electrodes. For the sake of completeness, the appendix lists some other examples of octupoles based on 
simple distributions of point, line and ring charges.

\section{Optimization of some example structures}

In this section we study various electrode arrangements and obtain the geometric factors $\gamma$ (eq. (19)) and $\mu$ (eq. (20) in each case. We optimize the most promising designs by adjusting electrode positions and relative sizes in order to maximise both factors.

In all the configurations to be discussed, we choose the $z$ axis along the line where the twin traps are situated, the $x-z$ plane is parallel to the planes containing sets of electrodes, and hence these planes are separated along the $y$-direction.

The geometric factors $\gamma$ and $\mu$ were obtained by the numerical method described in section 3.2 To obtain $\mu$ the r.f. electrodes were assigned a large voltage and the second derivative of $V(x, y, z)$ was calculated along the principal axes of the quadrupole perpendicular to the $z$ axis. In most cases these were the $x$ and $y$ directions (giving $\mu_{x}$ and $\mu_{y}$ ). Where this is not the case the radial geometrical parameters are denoted by $\mu_{x^{\prime}}$ and $\mu_{y^{\prime}}$. Where $\mu_{x}$ and $\mu_{y}$ differ, a component of the oscillating quadrupole potential lies along $z$, hence trapped ions would experience axial micromotion and the associated pseudopotential would contribute to their confinement along the $z$ direction, as discussed at the end of section 2.2

Since the numerical solution of Laplace's equation can be slow, we first gained some general insights by analytic methods. We roughly modelled the electrodes as a set of line charges, and wrote down the complete potential function. This permits $\gamma$ to be found as a function of various parameters, such as the line charges and their positions, and it can be maximised analytically. Results from such analyses gave a starting point for the electrode structures used in the numerical studies.

In order to know what value of $\gamma$ and $\mu$ one might hope for, we numerically calculated these parameters for two simple cases. These can be regarded as a 'standard' against which the results for our various electrode structures can be measured. As a standard for $\mu$, we used four cylindrical electrodes of radius $0.41254 a$ with axes aligned along the $z$ axis, and centred at the corners of a square of side $2 a$. This arrangement gave $\mu=0.20$. Electrodes of radius $0.3 a$ centred at the same positions gave $\mu=0.15$.

To obtain a standard value for $\gamma$, an electrode structure loosely based on the equipotentials of the axially symmetric octupole in equation [29 was used. This consisted of three toroidal electrodes centred on the $z$ axis at $z=0$ and $z= \pm 0.643 a$, with two spherical "end-cap" electrodes of radius $0.35 a$ at $z= \pm a$. The cross-section radii of the torii tubes and the radii of the centres of the tubes were $0.2 a$ and $a$ respectively for the $z=0$ torus and $0.2 a$ and $0.766 a$ respectively for the $z= \pm 0.643 a$ torii. This configuration gave $\gamma=0.133$.

\subsection{8 d.c. electrodes in 2 planes, 2 r.f. electrodes}

Let us first consider structures consisting of rod d.c. electrodes placed at a distance $p$ above and below the plane containing the r.f. electrodes. This "sandwich" arrangement is shown in figure $4 \mathrm{a}$ ). It is based on construction (E) of section 4.2 All the d.c. electrodes are at the same voltage.

Plan views of the two d.c. electrode structures tried are shown in figures $4 \mathrm{~s}$ and 5 We let $a$ set the overall distance scale, and then there are 4 parameters $(b, d, p, r)$ and 2 constraints. The octupole configuration was found by fixing $r$ and $b$, and then adjusting $p$ and $d$. 
a)

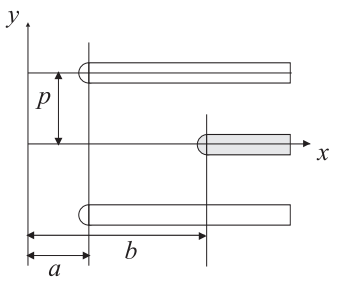

b)
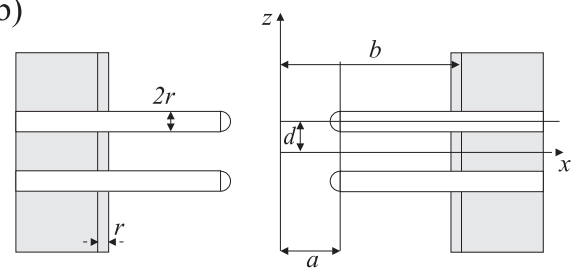

Fig. 4. Views of the electrode structure with 4 d.c. electrodes in each of two planes, and 2 r.f. electrodes. a) cross-section in the $x-y$ plane showing the sandwich structure. The r.f. electrodes are filled grey. b) View looking down on the $x-z$ plane from above.

The equipotential along the r.f. electrodes tends to prevent an octupole being produced at the origin. We found that it was necessary to restrict the influence of this equipotential by keeping $p$ small. Since $p$ must be larger than the electrode diameter this means that $r$ must not be too large.

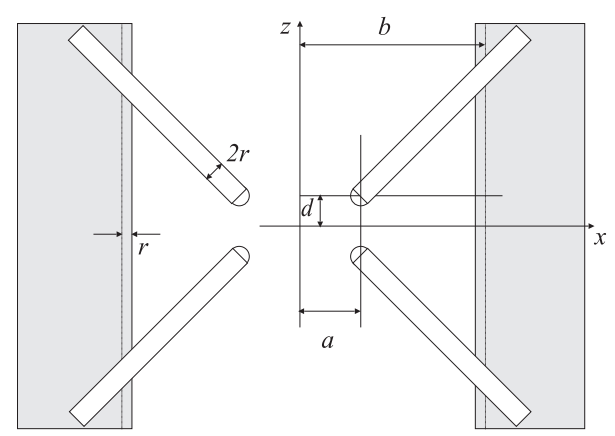

Fig. 5. A view of the electrode structure with 45 degree rod electrodes having hemispherical ends centred at $( \pm a, \pm p, \pm d)$. The r.f. electrodes have thickness $2 r$, and lie in the $y=0$ plane. The end of these electrodes are hemicylinders of radius $r$ and centered on $x= \pm b, y=0$. The grey fill represents the r.f. electrodes.

Results of the calculations for both structures are presented in table 1 The two cases produce similar values of $\gamma$ and $\mu$, those (labelled 1.1) for the structure shown in fig. 固 being

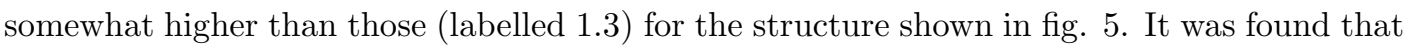
the r.f. electrodes had to be placed well back (a large value for $b$ ) in order to get a high value for $\gamma$.

Results 1.1 and 1.2 show that the value of $\mu$ decreases as $b$ is reduced. This is because $p$ and $d$ also decrease, with the result that the influence of the r.f. electrodes is screened by the d.c. electrodes. 
The geometry shown in figure 4 provides greater screening of the r.f. electrode by the d.c. electrodes, compared to that shown in figure 5 and this is probably the main reason why it provides the higher $\gamma$.

Table 1. Values of the distance parameters $r, b, p, d$ which give an octupole for three example electrode structures, with the distance to an electrode surface $\rho$ and the calculated geometric factors $\gamma$ and $\mu$ in each case. Results 1.1 and 1.2 are for electrode configurations of the form shown in figure 4 Result 1.3 is for an electrode configuration like that shown in figure 5

\begin{tabular}{|l|l|l|l|l|l||c|c|c|}
\hline Result & $r$ & $b$ & $p$ & $d$ & $\rho$ & $\gamma$ & $\mu_{x}$ & $\mu_{y}$ \\
\hline units & $(a)$ & $(a)$ & $(a)$ & $(a)$ & $(a)$ & $10^{-3}$ & $10^{-3}$ & $10^{-3}$ \\
\hline 1.1 & 0.30 & 6.30 & 1.94 & 2.01 & 2.67 & 26 & 6.40 & 6.45 \\
1.2 & 0.37 & 3.34 & 1.16 & 1.28 & 1.63 & 18 & 3.44 & 3.77 \\
\hline 1.3 & 0.26 & 4.95 & 1.20 & 0.88 & 1.53 & 15 & 4.80 & 3.14 \\
\hline
\end{tabular}

\subsection{0 d.c. electrodes in a plane, 4 r.f. electrodes}

This structure consists of 10 d.c. electrodes in the $y=0$ plane, and 4 r.f. electrodes in two planes parallel to this. It has reflection symmetry in $x-y, x-z$ and and $y-z$ planes. The d.c. electrodes are cylindrical, with hemispherical ends centred at $x= \pm a$. Figure 6 shows a cross section in the $x-z$ plane for positive $x$.

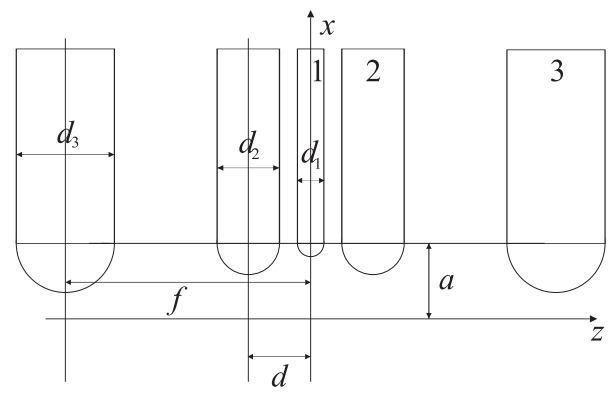

Fig. 6.10 d.c. electrodes, 2 r.f. electrodes: A cross section view of the d.c. electrodes in the $x-z$ plane with $y=0$ for positive $x$. The trap centre is at the intersection of the $\mathrm{x}$ and $\mathrm{z}$ axes. The electrode structure is repeated for negative $x$ by reflection in the $z-y$ plane.

Each r.f. electrode is effectively a semi-infinite plane, with its edge parallel to the $z$ axis. The $y$ position of the r.f. electrodes is $\pm p$, and the $x$-position is $\pm(a+t)$, hence $t$ gives the retraction of the r.f. electrodes. Where $p=1.03$ the r.f. electrode had thickness $a / 4$, otherwise the thickness was negligible. For the finite thickness cases, the edge of the electrode is hemicylindrical, and $p$ and $t$ give the position of the axis of the hemicylinder. An electrode structure with $p=1.03 a$ and $t=a$ is illustrated in figure 6 .

The voltage on electrode $3\left(\mathrm{~V}_{3}\right)$ was set to $1.4 \mathrm{~V}$ in all the calculations in this section. With a fixed geometry our free parameters are the voltages on electrodes 1 and $2\left(\mathrm{~V}_{1}\right.$ and $\mathrm{V}_{2}$ respectively). These were adjusted to fulfill the octupole condition, using a two-dimensional Newton-Raphson method in order to find the solution with few trials. Then the values of $\beta$, $E_{\max }$ and $\gamma$ were calculated. Adjustments were then made to $f, d$ and $d_{3}$, and the calculation repeated. A selection of positions and their corresponding values of $\gamma$ are shown in table 2 The nearest electrode surface distance $\rho=0.825 a$ in all cases. 

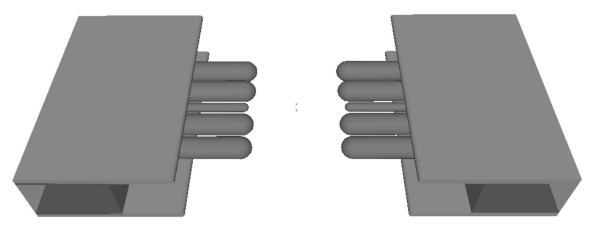

Fig. 7. A perspective view of the electrodes for r.f. electrodes positioned at $p=1.03 a$ and $t=a$. The 10 cylindrical electrodes have d.c. voltages applied to them. r.f. voltages are applied to the planar electrodes. Also shown are insulating spacers which hold the electrodes in place.

The initial choice of electrode diameters was $d_{1}=0.35 a, d_{2}=0.825 a, d_{3}=1.3 a$, based on the indications from our initial line charge calculations. Using these diameters, CPO calculations were carried out with no r.f. electrodes for several values of $f$ and $d$. The optimal values were found to be $f=3.25 a$ and $d=0.825 a$.

We then introduced the r.f. electrodes. With the r.f. electrodes far away from the d.c. electrodes $(p=2 a$ and $4 a$ ) the maximum field is found between the d.c. electrodes, hence varying $f$ makes little difference to the value of $\gamma$. This is because an increase (decrease) in $\beta$ is countered by an increase (decrease) in $E_{\max }$. Cases 2.1 and 2.2 in table 2 illustrate this. For large $d$, the maximum field is found between electrodes 2 and 3 , hence as $d$ is reduced, $E_{\max }$ decreases. For small $d$, the maximum field is found between electrodes 1 and 2 , and then as $d$ is reduced, $E_{\max }$ increases. This behaviour is shown in figure 8

Table 2. Results from CPO calculations. Results 2.1-2.6 are for the structures described in section 5.2 (c.f. figures 6 6), results 3.1-3.3 are for the inverted structures described in section [5.3 All the cases listed have $d=0.825 a, d_{1}=0.35 a, d_{2}=0.825 a, V_{\mathrm{rf}}=0$ and $V_{3}=1.4 \mathrm{~V}$. The table illustrates effects of changing the values of $f, d_{3}, p$ and $t$.

\begin{tabular}{|l|l|l|l|l|l|l||c|c|c||c|c|}
\hline Result & $f$ & $d_{3}$ & $p$ & $t$ & $V_{1}$ & $V_{2}$ & $10^{4} \beta$ & $E_{\max }$ & $\gamma$ & $\mu_{x}$ & $\mu_{y}$ \\
\hline & $(\mathrm{a})$ & $(\mathrm{a})$ & $(\mathrm{a})$ & $(\mathrm{a})$ & $(\mathrm{V})$ & $(\mathrm{V})$ & $\left(\mathrm{V} / a^{4}\right)$ & $(\mathrm{V} / a)$ & $10^{-3}$ & & \\
\hline 2.1 & 3.25 & 0.65 & 2 & 1 & 0.4605 & 0.2156 & 816 & 1.7 & 27 & 0.121 & 0.148 \\
2.2 & 2.513 & 0.65 & 2 & 1 & 0.6657 & 0.2501 & 1421 & 2.6 & 31 & 0.111 & 0.147 \\
2.3 & 3.25 & 0.413 & 4 & 3 & 0.6392 & 0.5065 & 500 & 1.9 & 14 & 0.096 & 0.110 \\
2.4 & 3.25 & 0.65 & 1.03 & 0 & 0.0973 & -0.0407 & 519 & 9 & 3.3 & 0.053 & 0.068 \\
2.5 & 3.25 & 0.65 & 1.03 & 1 & 0.3085 & 0.0650 & 764 & 9 & 4.8 & 0.030 & 0.037 \\
2.6 & 1.9 & 0.413 & 1.03 & 1 & 0.5218 & -0.0055 & 1819 & 5 & 20 & 0.053 & 0.069 \\
\hline 3.1 & 3.25 & 0.65 & 2 & 1 & 6.183 & -1.207 & 506 & 55 & 5.0 & 0.150 & 0.152 \\
3.2 & 3.25 & 0.65 & 1 & 1 & 0.3936 & -0.2378 & 1387 & 15 & 6.2 & 0.046 & 0.043 \\
3.3 & 3.25 & 0.65 & 2 & 3 & 4.969 & -0.3090 & 86 & 32 & 10 & 0.027 & 0.030 \\
\hline
\end{tabular}

When the r.f. electrodes are close to the d.c. electrodes $(p=1.03 a)$, the maximum field is found between the d.c. electrode 3 and the r.f. electrodes. This field can be reduced by reducing $d_{3}$. $\beta$ increases as $f$ is reduced, hence $\gamma$ can also be increased by reducing $f$ until the maximum field is found between the d.c. electrodes. Figure 9 shows this behaviour for structures with $p=1.03 a$ and $t=0$, and the net effect is illustrated by results $2.5,2.6$ in table 2

We also examined the effect of making electrode 3 extend further along the $z$ direction. It was replaced by a planar electrode of thickness $d_{3}$ with edges rounded off by hemicylinders, located such that the edge nearest the origin was situated at the same place as the cylindrical 

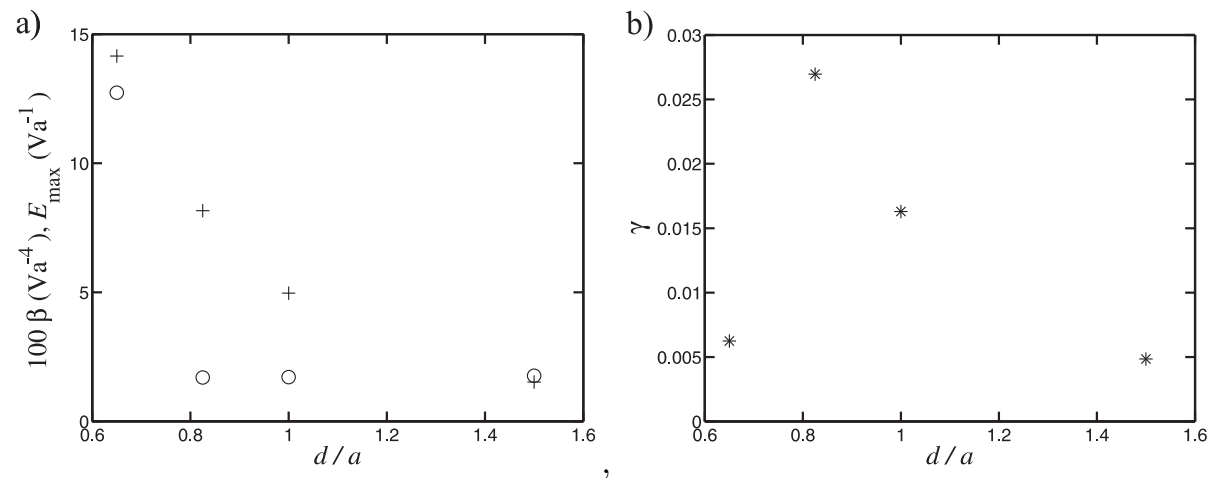

Fig. 8. The effect on $\beta, E_{\max }$ and $\gamma$ of adjusting $d$ for result 2.1 in table $2100 \beta$ is represented by + , and $E_{\max }$ by unfilled circles. If the largest field lies between the first and second electrodes, then reducing $d$ increases both $\beta$ and $E_{\max }$, and $\gamma$ drops. Where this is not the case, the increase in $\beta$ causes an increase in $\gamma$.

a)

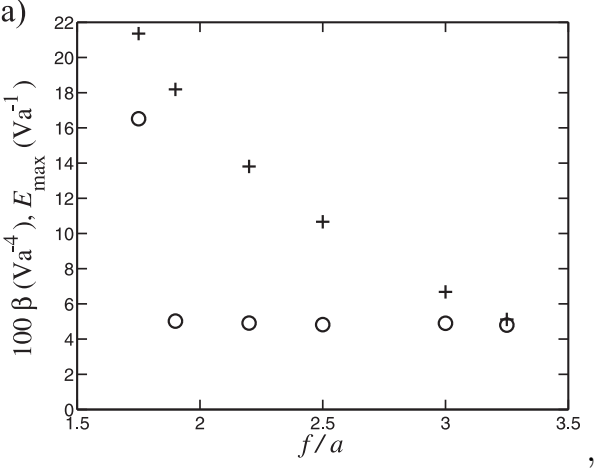

b)

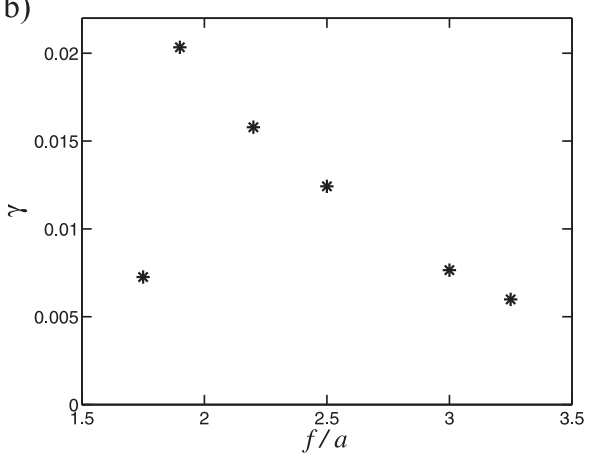

Fig. 9. The effect on $\beta, E_{\max }$ and $\gamma$ of adjusting $f$, with $d=0.825 a, d_{1}=0.35 a, d_{2}=0.825 a$, $d_{3}=0.825 a$, r.f. electrodes at $p=1.03 a, t=0.100 \beta$ is represented by + , and $E_{\text {max }}$ by circles. Reducing $f$ has the effect of increasing $\beta$. At larger $f$, the maximum field is found between the r.f. electrodes and the d.c. electrodes, hence we can increase $\gamma$ by decreasing $f$. After reaching the point where the largest field is found between the d.c. electrodes, $\gamma$ is much reduced for further reduction of $f$. 
electrode in the other results. This had little effect on $\gamma$ or $\mu$.

Finally, we examined an example in which the d.c. electrodes are extended along the $z$ direction into planar shapes. This will reduce $\gamma$, but it also reduces the $z$-dependence of the a.c. part of the potential, therefore giving less micromotion along $z$. Each electrode had two flat surfaces parallel to the $x-z$ plane, and sides in the shape of hemicylinders having the same radius of curvature as before. The electrode centres were placed as in result 2.6 and they were extended along the $z$ direction until the gaps between them were $0.1 a$. This arrangement gave $\gamma=2 \times 10^{-3}$, i.e. a factor 10 reduction compared to result 2.6.

\subsection{0 d.c. electrodes in two planes, 2 r.f. electrodes}

This arrangement is like the previous one, but with the d.c. and r.f. electrode positions swapped. It consists of 2 planar r.f. electrodes of thickness $0.5 a$ lying in the $y=0$ plane. These are bounded by hemicylindrical edges centred at $x= \pm(a+t)$. The d.c. electrodes lie in two planes at $y= \pm p$, each containing two sets of five d.c. electrodes (with $f, d$ and electrode diameters the same as result 2.1 in table 21). Results for these arrangements are shown in table 2 as results 3.1-3.3. The values of $\gamma$ and $\mu$ are similar to those in section 5.2 Some improvement in $\gamma$ would be expected through optimisation of the relative positions of the d.c. electrodes. This has not been carried out in obtaining results 3.1-3.3.

\subsection{Low Aspect Ratio Electrode Configurations}

Let $w$ be the total thickness, i.e. depth in $y$, of the electrode structure (c.f. figure 10). We define the aspect ratio $g=w / \rho$. The three-layer trap designs considered in sections 5.15 .2 and 5.3 have $g>1$. Though microfabrication techniques are available which could be used to construct traps with $\rho \leq 100 \mu \mathrm{m}$ and $g>1$ [26], many methods are limited to a maximum structure thickness of around $20 \mu \mathrm{m}$ [16, 27. This means that traps may have a low aspect ratio, $g<1$.

We studied structures similar to those of section 5.2 but now with $g<1$. To speed the calculation process we used cuboid shapes for the electrodes (see figures 10 and 11). The d.c. electrodes had width in $z$ of $d_{1}=d_{2}=d_{3}=w$, and were separated by gaps of $w / 2$ (hence $d=3 w / 2, f=3 w)$. The thickness of the electrodes in $y$ was $w / 5$. With a cuboid shape the electric field diverges near the electrode edges. In order to calculate $\gamma$ and $\mu$ we need to make some reasonable assumption to handle this. Our aim was to use the calculation to indicate approximately what would happen if in fact the electrode edges were smoothed. To this end we calculated electric field values at $(x, y, z)=(\rho, 0,0),\left(\rho+w / 5,0, d_{1} / 2\right),(\rho+t+w / 5, w / 10,0)$ and at similar places around the other electrodes, and took $E_{\max }$ to be the largest of these.

Results for structures with $g=0.4$ and $g=0.2$ are shown in figure 12 We found that retracting the r.f. electrodes (i.e. using $t>0$ ) gave a useful increase in both $\gamma$ and $\mu$. As the aspect ratio decreases, $\gamma$ falls rapidly (and $\mu$ less rapidly).

\subsection{Two-Layer Electrode Structures.}

The most natural configuration for producing a radial r.f. quadrupole in the $x-y$ plane consists of electrodes placed at four corners of a square. Equal r.f. voltages are applied to diagonally opposite electrodes. To produce an octupole by such an arrangement, one or more of these electrodes must be segmented. Choosing the structure to have 2-fold rotational symmetry about the $z$-axis, and reflection symmetry in the plane $z=0$, there remain 3 


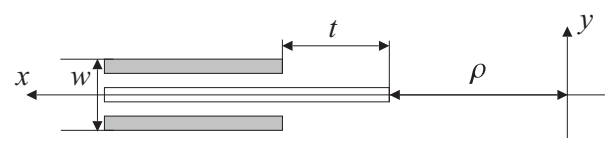

Fig. 10. A cross-section view of a low aspect ratio electrode structure for positive $x$ showing the definition of the parameters $w$ and $t$. The r.f. electrodes are represented by grey fill. The electrodes for negative $x$ are obtained by a reflection in the $x=0$ plane.
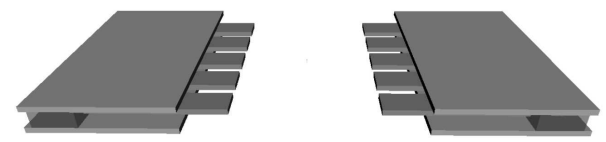

Fig. 11. Perspective view of a low aspect ratio electrode structure with $g=0.4$ and $t / \rho=0.6$.

a)

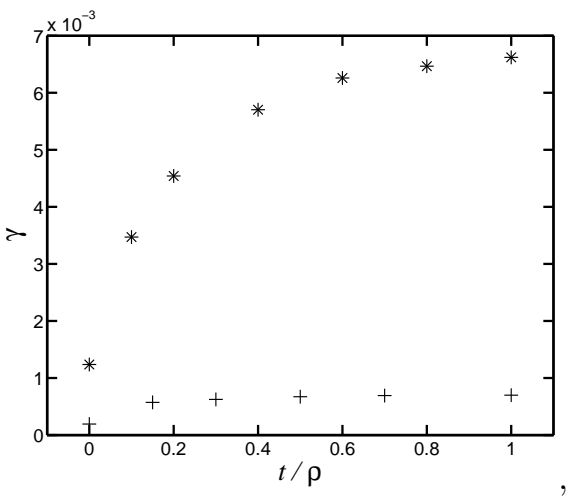

b)

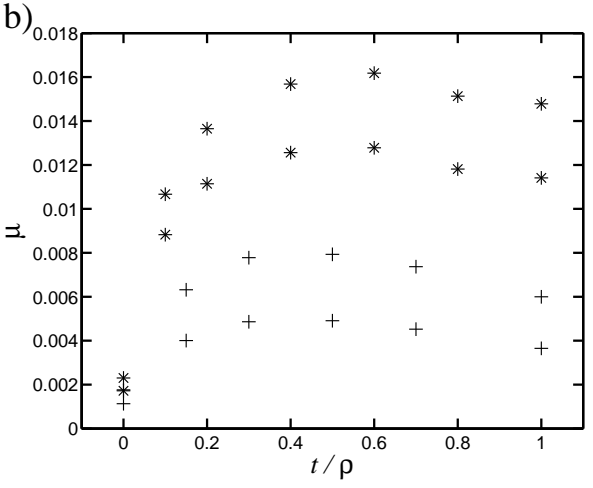

Fig. 12. Values of a) $\gamma$, b) $\mu$ for low aspect-ratio traps, as a function of the distance the r.f. electrodes are retracted with respect to the d.c. electrodes. Values for $g=0.4$ structures are marked by $*$, and values for $g=0.2$ are marked with a + . For a given $g$, the lower values of $\mu$ are $\mu_{y}$, the upper values are $\mu_{x}$. 
constraints. We chose to satisfy the constraints by adjusting voltages rather than by accurately placing electrodes, and therefore two diagonally opposite electrodes must be split into seven segments.

a)
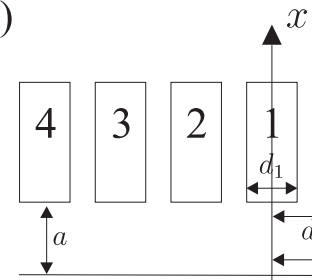

b)

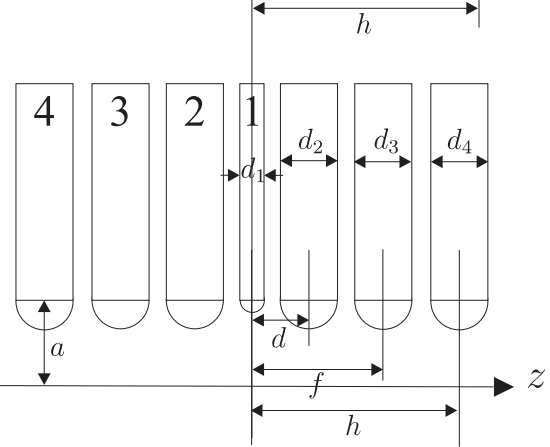

Fig. 13. The segmentation of the d.c. electrodes used in the two-layer electrode structures for a) cuboid electrodes and b) cylindrical electrodes. For cases where cuboidal electrodes were used, the electrode widths $d_{1}$ to $d_{4}$ were equal, and the separation between electrodes was $d_{1} / 2$. For structures with cylindrical electrodes, $d_{2}=d_{3}=d_{4}=2.36 d_{1}$.

The d.c. electrode segmentations used in the results presented below are shown in figure 13 The cuboid electrode structures consist of electrodes of equal width $\left(d_{1}=d_{2}=d_{3}=d_{4}\right)$, equally spaced by $d_{1} / 2$ (hence $d=3 / 2 d_{1}, f=3 d$ and $h=9 / 2 d$ ). The cylindrical electrode structures were made up of cylinders parallel to the $x$ axis with hemispherical ends. The diameters of the cylinders were $d_{2}=d_{3}=d_{4}=2.36 d_{1}$. The axes of the cylinders were positioned at $d=2.36 d_{1}, f=5.43 d_{1}$ and $h=8.43 d_{1}$. The hemispherical ends of the cylinders were centred on $x=a$.

The r.f. electrodes consist of extended planes of thickness $w_{\mathrm{rf}}$. For structures with cuboid electrodes, the end of the r.f. electrodes were placed at $x= \pm(a+t)$. For structures with cylindrical d.c. electrodes, the r.f. electrodes had hemicylindrical ends of radius $w_{\mathrm{rf}} / 2$ with axes parallel to the $z$ axis at $x= \pm(a+t)$.

The voltage on d.c. electrode 4 was set to $1.4 \mathrm{~V}$, and the r.f. electrode voltage was set to $0 \mathrm{~V}$. Numerical calculations were performed for a range of values of $d_{1}, t$ and $g$, as defined in figures 13 and 14. Example results of these calculations are presented in tables 3 and 4

We found that the width $d_{1}$ of the central electrode had a strong effect on $\gamma$ and a smaller effect on $\mu$ (see figure 15 and c.f results $\{4.5,4.6\},\{4.9,4.10\}$ ). The optimum value of $d_{1}$ for producing a d.c. octupole was found to be around $0.4 \rho$, for all values of $g$. This is comparable with the value used for the three layer electrode structures in section 5.2 which was obtained 
a)

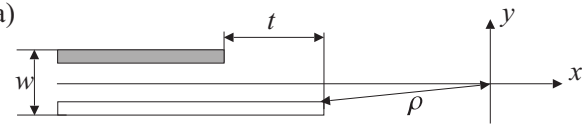

b)

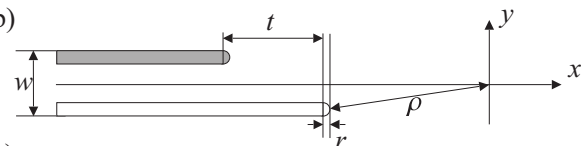

c)

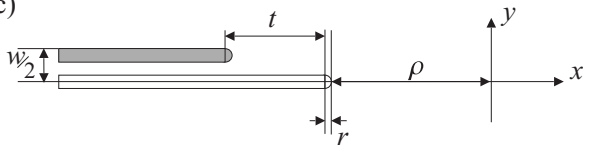

Fig. 14. Profiles of the two-layer electrode structures for negative $x$ viewed in the $x-y$ plane. The r.f. electrodes are represented by grey fill. a) is the structure used to obtain results 4.1 to 4.12 in table $3 \mathrm{~b}$ ) was used for results 4.13 to 4.15 , and c) for results 4.16 and 4.17 . The aspect-ratio of the trap was defined by $g=w / \rho$. The structure is repeated for positive $x$ by a rotation of $180^{\circ}$ about the $z$ axis.

by examining the equipotentials of line-charge calculations. The monotonic increase in $\mu$ with $d_{1}$ may be ascribed to an increased influence of all of the d.c. electrodes.

a)

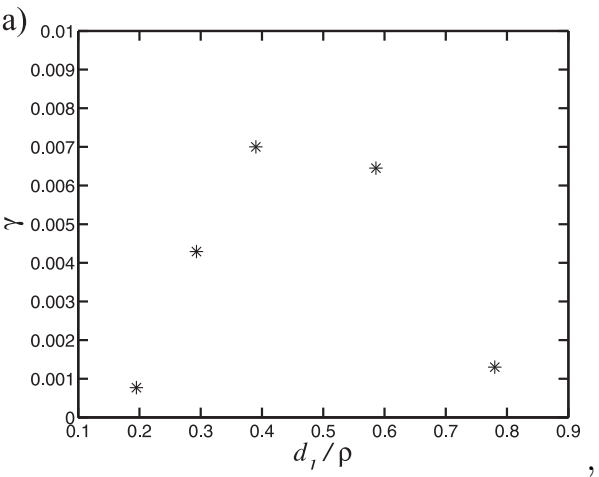

b)

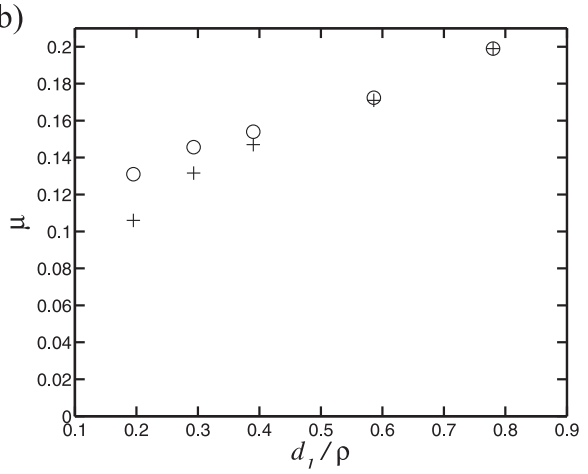

Fig. 15. The effect on a) $\gamma$ and b) $\mu$ of adjusting the width of the central electrode for a two-layer electrode structure having $g=2$ and $t=0 . \mu_{x^{\prime}}$ is represented by unfilled circles and $\mu_{y^{\prime}}$ by + .

Figure 16] shows the effect on $\gamma$ and $\mu$ of changing the aspect ratio of the trap. For $g<1$, both parameters increase with $g$. At higher $g, \mu$ continues to increase with $g$ while $\gamma$ decreases, presumably due to the reduced shielding of the r.f. electrodes by the d.c. electrodes.

We found that, for $g=2$, retraction of the r.f. electrodes had no significant effect on $\gamma$, but reduced $\mu$ somewhat (results $\{4.1-4.3\}$ ). When $g=0.4$ retraction allows a small increase in $\gamma$ with a drop in $\mu$ (results 4.7,4.8). When $g=0.24$, increasing $t$ from 0 to $\rho / 2$ increased $\gamma$ by $40 \%$ with little effect on $\mu$ (results $4.10,4.11$ ).

We examined the structure shown in figure 14k, with the d.c. electrodes placed in the $y=0$ plane, with a view to finding out whether it would produce a higher $\gamma$. However, comparison of results 4.17 and 4.15 suggest that it has the reverse effect. Since $\mu$ decreases also there is nothing to be gained by adopting this structure. 
a)

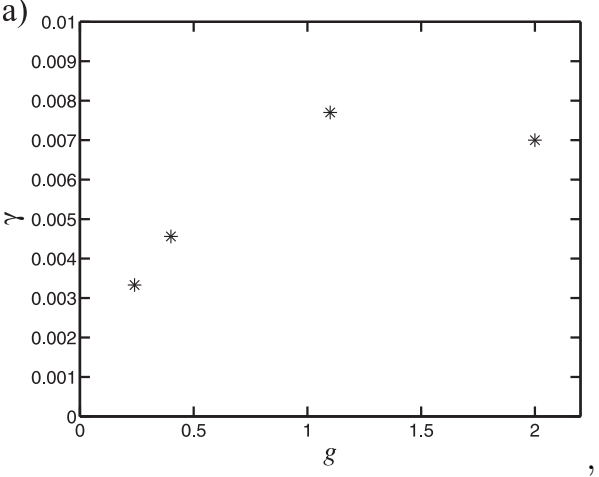

b)

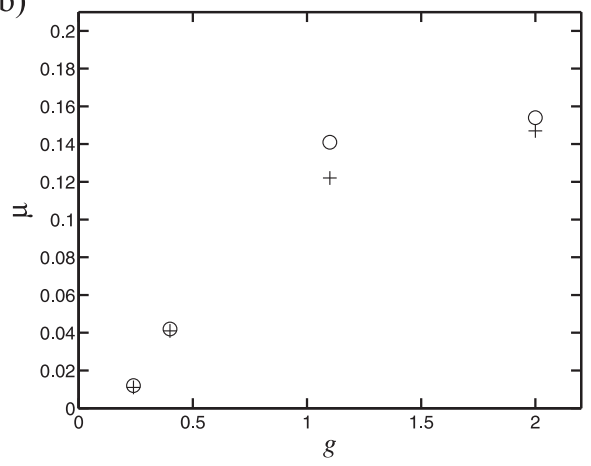

Fig. 16. The effect on a) $\gamma$ and b) $\mu$ of adjusting the aspect ratio $g=w / \rho$ for a two layer electrode structure. In b), the values of $\mu$ plotted are those along the $x^{\prime}$ (represented by + ) and $y^{\prime}$ (represented by $\circ$ ) axes of the quadrupole. The values of $d_{1}$ for these cases are similar but not identical $(0.39,0.47,0.4$ and $0.4 \rho$ from left to right on the plots).

\subsection{Planar or near-planar arrangments}

We considered cases where all the electrodes of the twin-trap system lie in a single plane (figure 17), and a simple 'railway track' arrangement in which rod-shaped r.f. electrodes run orthogonal to a set of simple rod-shaped d.c. electrodes (figure 18). The simplicity of such designs gives obvious advantages from the point of view of microfabrication, but we find that the penalty in terms of reduced factors $\gamma$ and $\mu$ is severe. These results are presented in table 5.

The voltages on these structures are to be adjusted to produce a trapping region centred outside the electrode structure. Therefore they lack one reflection symmetry, and there are 6 constraints if we require an octupole (construction (G) of section 4.2). To satisfy all these constraints by adjusting voltages would require 7 d.c. voltages (in addition to the d.c. ground set by the r.f. electrodes) and therefore a large number of electrodes. In order to reduce the number of electrodes, we chose to examine structures such that the hexupole moment may be small but non-zero. We adjusted the 5 voltages labelled A-E in figures 17 and 18 so as to cancel the quadrupole terms and $\partial V / \partial y$ and $\partial^{3} V / \partial y^{3}$, but we did not constrain $\partial^{3} V / \partial x^{2} \partial y$ or $\partial^{3} V / \partial z^{2} \partial y$.

To find the condition where the d.c. hexupole/octupole is centred at the same height as the r.f. quadrupole, we proceeded as follows. First the r.f. electrodes were set to zero, and the d.c. voltages were adjusted to produce a hexupole/octupole at a few different heights. Next, the r.f. electrodes were allowed to go to \pm 1 and the quadrupole position identified. We could then make a good first guess of the right d.c. voltages to get the desired coincidence, and the solution was found iteratively.

\section{Manufacturing imprecision and stray electric fields}

Throughout the above we relied on symmetries in the electrode structure in order to achieve the desired electric potential function. It is necessary to check whether there might be a high sensitivity to manufacturing imprecision and patch potentials which will in practice 


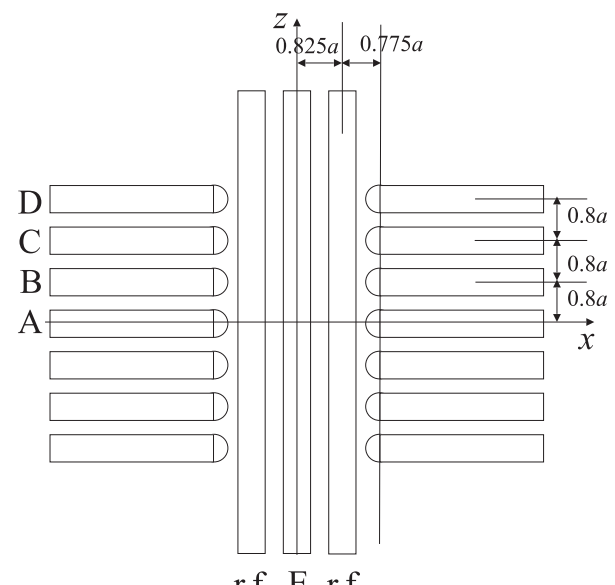

r.f. E r.f.

Fig. 17. The planar electrode arrangement (result 5.1). The cylindrical electrodes all lie in the same plane and have the same radius of $0.3 a$. The ends of the electrodes parallel to the $x$ axis are hemispherical.

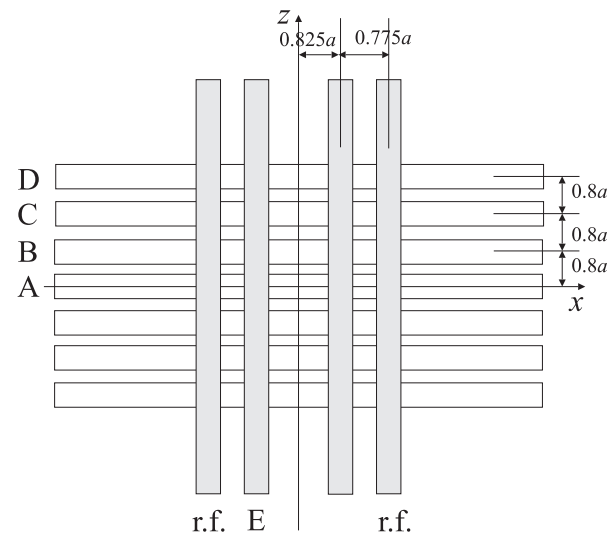

Fig. 18. Railway track electrode arrangement (result 5.2). The electrodes parallel to the $z$ axis are situated above those parallel to the $x$ axis by a distance of $a$. All electrodes are cylindrical, of radius $0.3 a$. 
break such symmetries. To this end we studied an example case: the electrode structure leading to result 2.2 in table 2 c.f. figure 6

In order to illustrate manufacturing imprecision, the electrode which has its spherical end centred at $(a, 0, d)$ was displaced by $0.05 \rho$ in the $z$ direction. The d.c. electrode voltages were then adjusted to satisfy $\partial^{2} V / \partial x^{2}=\partial^{2} V / \partial y^{2}=\partial^{2} V / \partial z^{2}=0$ at the centre of the trap, and the electric field $E_{z}(z=0)$ along the $z$ axis at the centre of the trap was calculated. The voltage on the displaced electrode was then adjusted to null this axial field, and the resulting potential shape $V(x, y, z)$ examined. This was repeated for displacements of the electrode in the $x$ and $y$ directions. The results are tabulated in table 6 The voltages are given such that

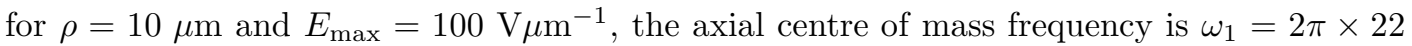
$\mathrm{MHz}$, and the radial centre of mass secular frequencies are $\omega_{x}=2 \pi \times 41 \mathrm{MHz}$ and $\omega_{y}=2 \pi \times 52$ $\mathrm{MHz}$, for calcium ions.

In this study we did not introduce any further electrodes, whereas in practice further electrodes would be present if the structure is part of a larger array, and such further electrodes could be used to cancel stray electric fields along $z$ such as the one obtained here. However by using the given set of electrodes we obtain an estimate of the precision required for the d.c. voltages, and an upper limit on the size of unwanted d.c. quadrupole terms which are introduced.

The values of $z_{c}$ in table 6 show that when the voltages are adjusted to cancel the quadrupole, then the stray electric field (owing to manufacturing imprecision) is large enough to cause a displacement of order $\rho$ of the ions, which would cause a problem. Comparing the values of $V_{2}$ in the 3rd and last columns of the table, however, it is seen that only a $\sim 5 \%$ adjustment in $V_{2}$ is needed to cancel this field. Since we are dealing with a $5 \%$ misplacement of the electrode, we infer that the required values of the electrode voltages are not especially sensitive to electrode misplacements. Equally, the figures show that the behaviour is sensitive to voltage inaccuracies, because without this same $5 \%$ voltage adjustment there would exist a severe problem.

To characterise the latter, argue as follows. In the presence of a field $E_{0}$ the centre of mass of the ions is displaced by approximately $z_{c}=q E_{0} / m \omega_{1}^{2}$, and at the octupole condition this evaluates to $z_{c}=E_{0} / 3 d^{2} \beta$ (using eq. (13)). This is a problem when the displacement is such that the potential hill introduced when $\alpha$ becomes negative does not appear in between the ion pair, so fails to separate the ions. Therefore the displacement is unacceptable if it exceeds approximately $d / 2$, so we require

$$
E_{0}<d^{3} \beta .
$$

In the example under discussion, $\beta \simeq 2.5 \times 10^{21} \mathrm{Vm}^{-4}$ and $d \simeq 1 \mu \mathrm{m}$, so the requirement is $E_{0}<2500 \mathrm{~V} / \mathrm{m}$. It is in practice easy to cancel fields of this magnitude, but in view of the fact that patch potentials and manufacturing imprecision can result in fields much larger than this, it may be necessary to characterise the electrode structure in situ in a vacuum system by transporting ions through it.

Next, consider the d.c. quadrupole terms. When the electrode structure lacks symmetry owing to its manufacturing imprecision, we can no longer assume mixed derivatives such as $\partial^{2} V / \partial x \partial y, \partial^{2} V / \partial y \partial x$ are zero, so we expect to get a d.c. quadrupole in the $x y$ plane when the electrodes are adjusted to satisfy the constraints $\partial V / \partial z=0, \partial^{2} V / \partial z^{2}=0$. In order to 
estimate the typical size of d.c. quadrupole caused by manfacturing imprecision, we left the electrode voltages at the values indicated in table [ without attempting any further nulling of quadratic terms, and examined the quadratic terms in the potential near the origin. These terms were of order $10^{9} \mathrm{Vm}^{-2}$. Under the conditions of the test, the radial confinement from the a.c. quadrupole is such as to achieve radial secular frequency around $45 \mathrm{MHz}$. We find that the influence of the d.c. quadrupole is small: it merely changes the radial secular frequencies to $\sim\left(45^{2} \pm 10^{2}\right)^{1 / 2} \simeq 45 \pm 1 \mathrm{MHz}$.

\section{Discussion}
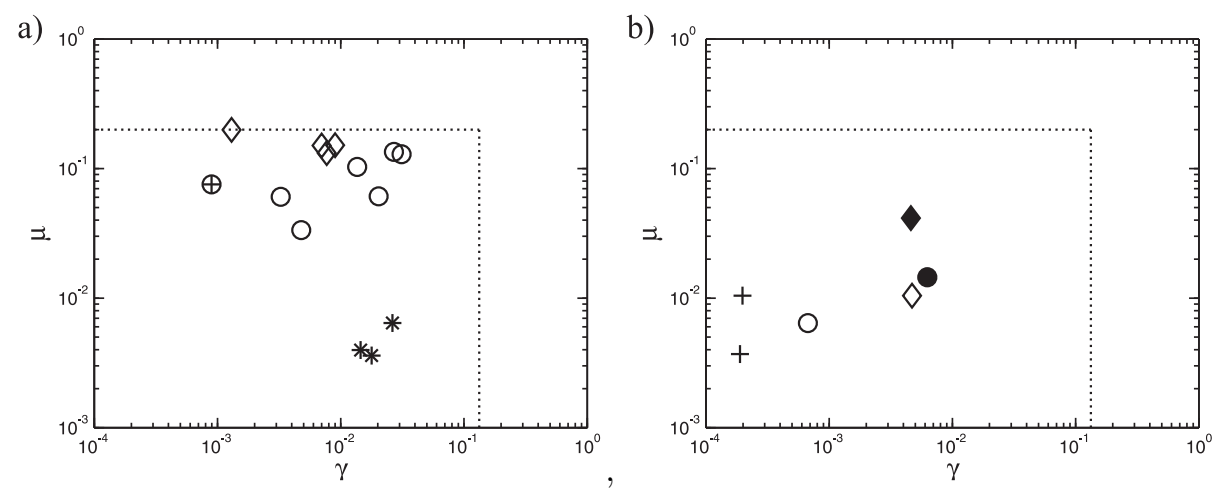

Fig. 19. Values of $\gamma$ and $\mu$ for the structures considered in the text. (a): high aspect ratio, $g \geq 1$; $*: 1.1-1.3, \circ: 2.1-2.6, \oplus: 3.1, \diamond: 4.1,4.2,4.7,4.14$. (b): low aspect ratio, $g \leq 0.75$; ○: 3-layer, $\diamond$ : 2-layer, + : 'planar' 5.1,5.2 $(g \simeq 0.65)$. The filled symbols represent results for $g \simeq 0.4$, the open symbols represent results for $g \simeq 0.2$. The dotted lines give the values which were obtained for the two 'standard' cases described in section 5 for comparison.

A summary of the values of $\gamma$ and $\mu$ obtained for the various structures we have considered is given in figure 19 We find that, with the exception of results 1.1-1.3, $\gamma$ is always smaller than $\mu$. This may be because we have not allowed the r.f. electrode to be divided. It presents a flat potential surface along the $z$ direction which tends to suppress the variation in potential which is needed for large $\gamma$. It may be possible to increase $\gamma$ without introducing multiple r.f. electrodes, by tailoring the shape of the edge of the r.f. electrodes to follow an equipotential produced by the d.c. octupole.

One might expect a competition between $\mu$ and $\gamma$ to be observed in the results, but there is little indication of this. The best structures give high $\gamma$ and high $\mu$ simultaneously.

Many of the optimizations studied, by adjusting the widths and placements of electrodes, gave useful improvements in $\gamma$ and/or $\mu$. This implies that a careful optimization of any chosen design is worthwhile.

Of the three-layer electrode structures we examined, those of the general form shown in figure 7 performed best. The highest $\gamma$ value $(\gamma \simeq 0.03$, result 2.2$)$, was obtained with $\mu$ close to the highest value, using a 3-layer structure of aspect ratio $g \simeq 3$.

At lower aspect ratio, $g<0.5$, the two-layer structures performed better than three-layer ones of the same $g$ (figure 19p). The larger value for $\gamma$ may be understood from the fact that when $g$ is small, $\gamma$ varies rapidly with $w$ because thicker electrodes can extend their 
influence on $V(x, y, z)$ over larger distances. For a given total depth $w$ of the whole structure, if there are $m$ layers and $m-1$ gaps all of the same thickness, then the thickness of each layer is $w /(2 m-1)$. Therefore a two-layer structure of given $g$ may be expected to give a similar value for $\gamma$ as a three-layer structure with $g$ larger by a factor $5 / 3$. This may be seen by comparing the values for a three-layer structure at $g=0.4$ in figure 12 with those for a two-layer structure at $g=0.24$, e.g. result 4.11 (and c.f. figure 19b). The two-layer structures also give larger values for $\mu$ at given $g$. This is in part for a similar reason, and also because the electrodes are more conveniently placed to realise a radial quadrupole. The two-layer structures of large aspect ratio satisfy the condition (21) at $q_{r}=0.3$ which means that, owing to their good radial confinement, it is not necessary to cancel d.c. quadrupole terms in the potential, but doing so remains useful in order to reduce the required r.f. voltages.

At $g=0.24$ the highest $\gamma$ value $(\gamma \simeq 0.005$, result 4.11) was smaller than the best overall by a factor 6 , and $\mu$ was reduced by an order of magnitude.

The planar or near-planar arrangements, cases 5.1 and 5.2, gave a further order of magnitude reduction in $\gamma$ compared to the two-layer low-aspect-ratio structure. Owing to this small $\gamma$ value, these structures satisfy the condition (21) so the cancellation of d.c. quadrupole terms is not necessary.

\section{Conclusions}

We have studied the general problem of separating or bringing together pairs of trapped ions, while keeping the frequencies of the normal modes of oscillation of the ions as high as possible. A simple picture of this is to say the central electrode introduces a potential hill which pushes the ions apart. However we have found that the distance between the ions can be small compared to the distance scale of the electrodes, even when the potential well is about to divide into two local minima. This means that the potential shape in the region between the ions is really a joint property of the whole set of electrodes. There are various contributions to the potential that have a quadratic dependence on position near the origin, and when the ions are being split or recombined these are balanced, so as to leave a quartic dependence.

The problem under consideration reduces to two main issues: radial confinement, and axial confinement at the point where it is weakest, which is to say when the axial potential is quartic. We found that axial confinement is the harder to achieve in large structures, and radial confinement in small ones. The size scale $\rho_{c}$ when the radial confinement becomes the limiting problem is of order microns. It is set largely by a length scale $L_{0}$ (equation (25)) which is determined purely by materials considerations, i.e. by the maximum electric field allowed on electrode surfaces. Since $L_{0}$ varies as $E_{\max }^{1 / 2}$ it will not change greatly for different materials or fabrication methods. Ion trap micro-fabrication methods being attempted at present are in the regime $\rho \gg \rho_{c}$.

We have studied a variety of electrode structures and characterised them mainly through the geometrical factors $\mu$ and $\gamma$ (equations (20) and (19)) which characterise the strength of r.f. quadrupole and d.c. octupole moments. Many of the structures exhibit $z$-dependence

\footnotetext{
${ }^{a}$ Methods have been suggested to approach the nanometre scale, such as the use of carbon nanotubes as electrodes. The use of very tight traps would require novel non-optical methods to achieve quantum logic gates, because the Lamb-Dicke parameter would be very small.
} 
in the oscillating part of the potential, and therefore micro-motion along the $z$ axis. This motion is found to have a small Matthieu $q$-parameter and is not expected to be a problem. We assumed reflection symmetries in the structures, and for one example case we examined the effects of manufacturing imprecision which broke the symmetry. This did not suggest any major problem with the design, in that the unwanted electric field owing to manufacturing error could be cancelled by small adjustments to the electrode voltages, and the unwanted quadrupole terms were small.

We found that for large aspect ratio, 3-layer designs gave the highest values of $\gamma$ and simultaneously almost the highest $\mu$. At low aspect ratio, 2-layer designs performed best at a given aspect ratio. Current efforts to fabricate trap arrays are focussed on distances of order $\rho \simeq 100 \mu \mathrm{m}$ and this suggests a low aspect ratio $g \simeq 0.2$ will be necessary if lithographic methods are used. In the future, however, there may be interest in $\rho \simeq 10 \mu \mathrm{m}$ and then $g \simeq 2$ will be available.

It is not surprising that the 3-layer designs offer tighter traps than the planar designs, but it is noteworthy that when considering the problem of separating ions, this is especially true. The factor $\simeq 150$ decrease in $\gamma$ (comparing case 2.2 in table 2 with case 5.1 in table 5 means that the planar design would need to be fabricated at a distance scale a factor $150^{1 / 3} \simeq 5$ times smaller in order to obtain the same motional frequency $\omega_{1}$ (equation (22)). If we assume heating rates scaling as $\rho^{-4}$, the heating rate in the latter case would be increased compared to the former by a factor $\simeq 800$. If instead one compares structures having the same number of phonons of heating per ion separation time (c.f. section 3.1), then the planar design leads to a motional frequency a factor $150^{0.387} \simeq 7$ lower.

In view of the greater ease of fabrication of planar structures, a sevenfold reduction of motional frequencies might be regarded as acceptable. However, for a quantum computer in which moving ions around and separating and combining strings of ions are essential ingredients to most logical operations, the separation/combination time may be the main limitation on the overall logic speed. To establish a definite design preference between a small planar structure and a larger layered structure, a greater understanding of the heating mechanism will be needed.

\section{Acknowledgements}

We thank D.N.Stacey, M.G.Blain and J.Fleming for helpful discussions. We thank D.Leibfried for information on the NIST trap dimensions.

This work was supported by the EPSRC, the Research Training and Development and Human Potential Programs of the European Union, the National Security Agency (NSA) and Advanced Research and Development Activity (ARDA) (P-43513-PH-QCO-02107-1).

\section{References}

1. D. J. Wineland, C. Monroe, W. M. Itano, D. Leibfried, B. E. King, and D. M. Meekhof. Experimental issues in coherent quantum-state manipulation of trapped atomic ions. J. Res. Natl. Inst. Stand. Technol., 103:259-328, 1998.

\footnotetext{
${ }^{b}$ We have carried out example numerical integrations of the equations of motion for a pair of ions undergoing
} controlled separation in order to gain evidence of this. 
2. A. M. Steane The ion trap quantum information processor. App. Phys., B64, 623-642, (1997)

3. M. Sasura, V. Buzek Cold Trapped Ions as Quantum Information Processors J. Mod. Opt., 49, 1593-1647 (2002)

4. F. Schmidt-Kaler, H. Hffner, M. Riebe, S. Gulde, G. P. T. Lancaster, T. Deuschle, C. Becher, C. F. Roos, J. Eschner and R. Blatt. Realization of the Cirac-Zoller controlled-NOT quantum gate. Nature, 422:408-411, March 2003

5. D. Leibfried, B. DeMarco, V. Meyer, D. Lucas, M. Barrett, J. Britton, W. M. Itano, B. Jelenkovic, C. Langer, T. Rosenband and D. J. Wineland. Experimental demonstration of a robust, highfidelity geometric two ion-qubit phase gate Nature, 422:412-415, March 2003

6. C. A. Sackett, D. Kielpinski, B. E. King, C. Langer, V. Meyer, C. J. Myatt, M. Rowe, Q. A. Turchette, W. M. Itano, D. J. Wineland, and C. Monroe. Experimental entanglement of four particles. Nature, 404:256-258, March 2000.

7. M. Riebe, H. Hffner, C. F. Roos, W. Hnsel, J. Benhelm, G. P. T. Lancaster, T. W. Krber, C. Becher, F. Schmidt-Kaler, D. F. V. James, and R. Blatt. Deterministic quantum teleportation with atoms. Nature, 429:734-737, 2004.

8. C. F. Roos, M. Riebe, H. Haffner, W. Hansel, J. Benhelm, G. P. T. Lancaster, C. Becher, F. Schmidt-Kaler, and R. Blatt. Control and measurement of three-qubit entangled states. Science, 304(1478), 2004.

9. D. Kielpinski, C.Monroe, and D. Wineland. Architecture for a large-scale ion-trap quantum computer. Nature, 417:709-711, June 2002.

10. A. M. Steane. Quantum computer architecture for fast entropy extraction. Quant. Inf. and Comp., 2:297-306, 2002. quant-ph/0203047

11. M. A. Rowe, A. Ben-Kish, B. DeMarco, D. Leibfried, V. Meyer, J. Beall, J. Britton, J. Hughes, W. M. Itano, B. Jelenković, C. Langer, T. Rosenband, and D. J. Wineland. Transport of quantum states and separation of ions in a dual rf ion trap. Quantum Information and Computation, $2(4): 257,2002$.

12. M. D. Barrett, J. Chiaverini, T. Schaetz, J. Britton, W. M. Itano J. D. Jost, E. Knill, C. Langer, D. Leibfried, R. Ozeri, and D. J. Wineland. Deterministic quantum teleportation of atomic qubits. Nature, 429:737-739, 2004.

13. W. K. Hensinger, S. Olmschenk, D. Stick, D. Hucul, M. Yeo, M. Acton, L. Deslauriers, C. Monroe, and J. Rabchuk. T-junction ion trap array for two-dimensional shuttling, storage and manipulation. quant-ph/0508097

14. Q. A. Turchette, D. Kielpinski, B. E. King, D. Leibfried, D. M. Meekhof, C. J. Myatt, M. A. Rowe, C. A. Sackett, C. S. Wood, W. M. Itano, C. Monroe, and D. J. Wineland. Heating of trapped ions from the quantum ground state. Phys. Rev., A61:063418, 2000.

15. L. Deslauriers, P. C. Haljan, P. J. Lee, K.-A. Brickman, B. B. Blinov, M. J. Madsen, and C. Monroe. Zero-point cooling and low heating of trapped cd ions. Phys. Rev. A, 2004. quant-ph/0404142

16. M. J. Madsen, W. K. Hensinger, D. Stick, J. A. Rabchuk, and C. Monroe. Planar ion trap geometry for microfabrication. Appl. Phys. B, 78:639-651, 2004.

17. M. Šasura and A. M. Steane. Fast quantum logic by selective displacement of trapped ions. Phys. Rev., A67(062318), 2003.

18. A. Sørenson and K. Mølmer. Entanglement and quantum computation with ions in thermal motion. Phys. Rev., A62(022311), July 2000.

19. D. Leibfried, R. Blatt, C. Monroe, D. J Wineland Quantum dynamics of single trapped ions. Rev. Mod. Phys., 75, 281-324, 2003

20. H. G. Dehmelt. Radiofrequency spectroscopy of stored ions. Adv. At. Mol. Phys, 3(53), 1967.

21. Prahdip K. Ghosh. Ion Traps. Clarendon Press, Oxford, 1995.

22. D. Cruz, J.P. Chang, and M. G. Blain. Field emission characteristics of a tungsten micro-electrical mechanical system device. preprint, ., 2004.

23. R. Gomer. Field Emission and Field Ionization. Harvard University Press, Cambridge, 1961.

24. Idaho National Engineering and Environmental Laboratory. Simion 3d version 7.0, 2000.

25. CPO Ltd. Charged particle optics programs. 
26. J.R. Reid and R.T. Webster. A $60 \mathrm{ghz}$ branch line coupler fabricated using integrated rectangular coaxial lines. IEEE, 2004.

27. M. G. Blain, L. S. Riter, D. Cruz, D. E. Austin, G. Wu, W. R. Plass, and R. Graham Cooks. Towards the hand-held mass spectrometer: design considerations, simulation, and fabrication fo micrometer-scaled cylindrical ion traps. International Journal of Mass Spectrometry, 236:91-104, 2004 .

\section{Appendix 1: Axial micromotion}

Consider the axial motion of an ion in the potential given in equation (14):

$$
V(0,0, z)=\alpha z^{2}+\beta z^{4}+\left(\alpha_{z} z^{2}+\beta_{r} z^{4}\right) \cos (\Omega t)
$$

where $\Omega$ is the frequency of the voltage applied to the r.f. electrodes, and we added an octupole term to the oscillating part in case that is important (it will turn out that it is not). For small departures from equilibrium, the equation of motion for ion $i$ of an $n$-ion chain (assuming the ions are confined strongly in the radial direction) is

$$
m \frac{d^{2} z_{i}}{d t^{2}}=-q\left(2 \alpha z_{i}+4 \beta z_{i}^{3}+\left(2 \alpha_{z} z_{i}+4 \beta_{r} z_{i}^{3}\right) \cos (\Omega t)\right)+\sum_{j} k_{i j}\left(z_{i}-z_{j}\right)
$$

where $z_{i}$ is the position of ion $i$, and $k_{i j}$ is the spring constant arising from the Coulomb term in the expansion of the ion-ion repulsion (this expansion has only been taken to first order in the ion separation).

When we sum the equations for each of two ions, and the ion-ion repulsion term drops out, leaving

$$
m\left(\frac{d^{2} z_{1}}{d t^{2}}+\frac{d^{2} z_{2}}{d t^{2}}\right)=-q\left(2 \alpha+2 \alpha_{z} \cos (\Omega t)\right)\left(z_{1}+z_{2}\right)+\left(4 \beta+4 \beta_{r} \cos (\Omega t)\right)\left(z_{1}^{3}+z_{2}^{3}\right) .
$$

Now substitute $z_{c}=\left(z_{1}+z_{2}\right) / 2$, and $z_{1}-z_{2}=d+\zeta$ where $d$ is the equilibrium separation of the ions and $\zeta$ represents excursions of the ions from their equilibrium separation. Equation (41) becomes

$$
m \frac{d^{2} z_{c}}{d t^{2}}=-2 q\left(\alpha+\alpha_{z} \cos (\Omega t)\right) z_{c}-q\left(\beta+\beta_{r} \cos (\Omega t)\right)\left(3(d+\zeta)^{2}+4 z_{c}^{2}\right) z_{c} .
$$

In the limit $\zeta, z_{c} \ll d$ we neglect the terms of order $\zeta d, \zeta^{2}$ and $z_{c}^{2}$ in the second term on the right hand side. The resulting equation can be transformed to the standard form of the Matthieu equation

$$
\frac{d^{2} z_{c}}{d \xi^{2}}+\left(a_{z}-2 q_{z} \cos (2 \xi)\right) z_{c}=0
$$

using the substitutions

$$
\xi=\frac{\Omega t}{2}, a_{z}=\frac{4 q}{m \Omega^{2}}\left(2 \alpha+3 \beta d^{2}\right), q_{z}=-\frac{4 q \alpha_{z}}{m \Omega^{2}},
$$

where we assume the d.c. octupole is such that $\beta d^{2} \sim \alpha$, but the a.c. octupole term can be neglected since $\beta_{r} \sim \alpha_{z} / a^{2} \ll \alpha_{z} / d^{2}$. The secular frequency associated with the solutions of 
equation (43) is given by

$$
\begin{aligned}
\omega_{c}^{2} & =\left(\frac{\Omega}{2}\right)^{2}\left(a_{z}+q_{z}^{2} / 2\right) \\
& =\frac{q}{m}\left(2 \alpha+3 \beta d^{2}+\frac{\left|q_{z} \alpha_{z}\right|}{2}\right) .
\end{aligned}
$$

This equation should be compared with (10). We can obtain (46) from (10) by the substitution

$$
\alpha \rightarrow \alpha^{\prime}=\alpha+\frac{1}{4}\left|q_{z} \alpha_{z}\right|
$$

The same result is obtained by treating the effect of the oscillating part of $V(0,0, z)$ using a static pseuodopotential.

In the electrode structures discussed in this paper, the oscillating part of the potential has a quadrupole form with axial coefficient always less than half the radial coefficients, $\left|\alpha_{z}\right|<(1 / 2)\left|\alpha_{r}\right|$. This means that if we choose the radial Mathieu $q$-parameter $q_{r}<0.3$, then $\left|q_{z}\right|<0.15$. In the most extreme case we find that to obtain $\alpha^{\prime}=0$ we require $\alpha \sim-0.04\left|\alpha_{r}\right|$. We find that this requires only a small adjustment to the voltages, compared to the values required for $\alpha=0$.

\section{Appendix 2: Basic octupole constructions}

We list some combinations of point and line charges that produce octupoles using a minimal number of separate points or lines.

\subsection{Systems of point charges}

8 point charges $q$ at the corners of a regular cube of side $2 a$ produce an octupole having cubic symmetry (30) with $\beta=7 q / 81 \sqrt{3} \pi \epsilon_{0} a^{5}$.

If we search for a system of 8 point charges all lying in a single plane $y=0$, the only solution is the one produced by the construction (32), (33). This is shown in figure 20k. In the positive quadrant there is a charge $q$ at $(x, z)=(a, d)$ and a charge $-f^{3} q$ at $(f a, f d)$. For example, $a=d$ gives $\beta=\left(-13 q / 128 \sqrt{2} \pi \epsilon_{0} a^{5}\right)\left(1-1 / f^{2}\right)$; the coefficients of $x^{4}, y^{4}$ and $z^{4}$ are then in the ratios $13:-12: 13$, and the potential is a combination of axial and $2 \mathrm{D}$ octupoles in the ratio $-24: 35$. When $d=a / \sqrt{2}$ then each set of 4 charges on its own produces a potential with $\partial^{2} V / \partial z^{2}(0,0,0)=0$, and the two sets as described produce equal and opposite $\partial^{2} V / \partial x^{2}(0,0,0)$. This gives $\beta=\left(-14 \sqrt{2 / 3} q / 81 \pi \epsilon_{0} a^{5}\right)\left(1-1 / f^{2}\right)$; the coefficients of $x^{4}, y^{4}$ and $z^{4}$ are in the ratios $26:-54: 56$. Similar remarks apply with $x$ and $z$ swapped when $d=\sqrt{2} a$.

\subsection{Systems of ring and line charges}

The potential produced by a semi-infinite straight line charge is $V=\left(\lambda / 2 \pi \epsilon_{0}\right) \ln (r+z)$, in a coordinate system such that the line charge lies on the negative $z$ axis and ends at the origin $\left(r=\left(x^{2}+y^{2}+z^{2}\right)^{1 / 2}\right)$.

An axially symmetric octupole (29) can be created by a single pair of identical ring charges on a common axis, whose separation is $\sqrt{2}$ times larger than their radius (shown in figure 20k). For charge per unit length $\lambda$ and radius $a, \beta=-14 \sqrt{2 / 3} \lambda / 81 \epsilon_{0} a^{4}$. This charge distribution 
can also be interpreted as a rotation about the $z$ axis of point charges at the 'special' location $x=z / \sqrt{2}$ which we identified in the preceeding section.

Addition of a further pair of rings allows a solution at any values of the ring separations, by adjusting the free parameter of the relative charge per unit length.

A set of 8 identical line charges, ending at the corners of a regular cube of side $2 a$, and extending outwards along the extended major diagonals, produces an octupole having cubic symmetry (30) with $\beta=-56 \lambda / 2592 \pi \epsilon_{0} a^{4}$, where $\lambda$ is the charge per unit length.

Next let us consider two sets of 4 line charges lying in two parallel planes (construction (E) of section 4.1). To reduce the number of parameters, we consider semi-infinite lines. We take the origin at the centre of symmetry, and orient axes so that the two planes are parallel to the $x-z$ plane. Apart from an overall scale factor, there are then three parameters which describe the layout: the angular coordinates of the end of one of the lines, which may be specified for example by spherical polar angles $\theta, \phi$, and the angle $\varphi$ between the line charge and the $z$-direction. This is shown in figure 20. Since we have three parameters $(\theta, \phi, \varphi)$ and two constraints (35), one parameter may be chosen arbitrarily and the others adjusted to find a solution. For example in the construction (E) we pick $\varphi$ and then adjust $\theta$ and $\phi$. We will list some example solutions. For convenience, we specify $\theta, \phi$ by giving the rectangular coordinates $(x, y, z)$ of the end of the line lying in the positive octant, with an arbitrary scale factor. Example solutions are $(x, y, z, \varphi)=(1,1,0,0),(1,1.434,0.578, \pi / 6),(1,1.799,1, \pi / 4)$, $(1,2.482,1.731, \pi / 3),(0,1,1, \pi / 2)$.

A set of 4 parallel infinite line charges passing through the corners of a square in a plane perpendicular to them (figure 20 ) produces a $2 \mathrm{D}$ octupole (31) with $\beta=\lambda / 8 \pi \epsilon_{0} a^{4}$, where the square has side $2 a$ (see figure 20,

A set of 4 infinite line charges passing along the sides of a cube as in figure 20: produces an octupole having $\beta=-3 \lambda / 4 \pi \epsilon_{0} a^{4}$, at the centre of the cube, where the cube has side $2 a$.

Next, consider sets of semi-infinite line charges all lying in a single plane. Let the plane be the $x-z$ plane and assume symmetry under reflections in the $x$ and $z$ axes. First suppose there is only a single line charge in the positive quadrant (so 4 line charges in total). The geometry has two parameters: the angle $\theta$ between the $z$ axis and a vector from the origin to the end of the line, and the angle $\varphi$ between the line itself and the $z$-direction. This suggests it might be possible to obtain $\partial^{2} V / \partial x^{2}=\partial^{2} V / \partial z^{2}=0$ by a good choice of $\theta$ and $\varphi$, but the pair of simultaneous equations has no solution. The construction (D) (eq. (33)) leads to an infinite set of solutions with 8 electrodes in two groups of four. The two groups have the same values of $\theta$ and $\varphi$; the charge per unit length on the outer group is greater than that on the inner group by a factor $f^{2}$. There are further solutions when the two groups have different angles. As an example case, shown in figure 20 $\mathrm{b}, \theta=\pi / 4, \varphi=\pi$ (line charges parallel to the $x$ axis and finishing on the line $x=z)$ gives $\beta=-\left(15 \lambda(16+11 \sqrt{2}) / 16 \pi \epsilon_{0}(1+\sqrt{2})^{4} a^{4}\right)\left(1-1 / f^{2}\right)$. 
a)

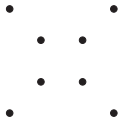

c)

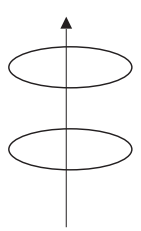

e)

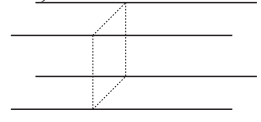

g)

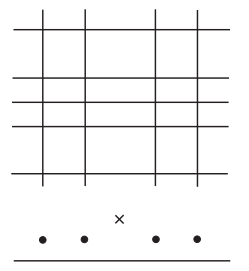

b)

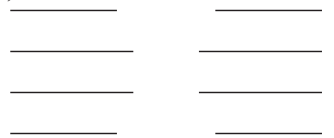

d)

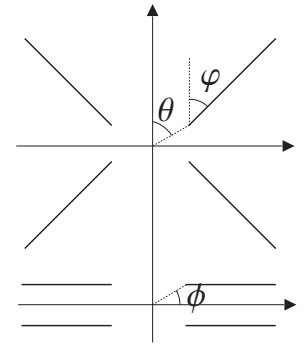

f)

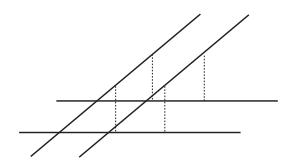

h)

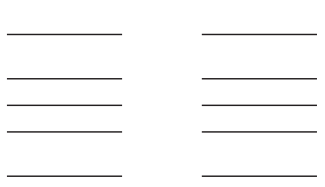

Fig. 20. Examples of octupole contructions using line charges. 
Table 3. Results from numerical calculations for the two-layer electrode structures. The value of $E_{\max }^{\mu}$ was calculated using an r.f. electrode voltage of $10^{6} \mathrm{~V}$. Results 4.1 to 4.12 use cuboid electrodes. Results 4.13 to 4.17 use cylindrical d.c. electrodes in the arrangement shown in figure 134.13 to 4.15 are for case (b) in figure 14 4.16 and 4.17 for for case (c) in figure 14]

\begin{tabular}{|l|l|l|l|l||c|c|c||c|c|c|}
\hline Result & $g$ & $w_{\mathrm{rf}}$ & $d_{1}$ & $t$ & $10^{4} \beta$ & $E_{\max }$ & $\gamma$ & $E_{\max }^{\mu}$ & $\mu_{x^{\prime}}$ & $\mu_{y^{\prime}}$ \\
\hline units & & $(\rho)$ & $(\rho)$ & $(\rho)$ & $\left(\mathrm{Va}^{-4}\right)$ & $\left(\mathrm{Va}^{-1}\right)$ & $\left(10^{-3}\right)$ & $\left(10^{6} \mathrm{Va}^{-1}\right)$ & \\
\hline 4.1 & 2 & 0.16 & 0.39 & 0 & -710 & 21 & -7.0 & 1.77 & 0.147 & 0.154 \\
4.2 & 2 & 0.16 & 0.39 & 0.39 & -728 & 23 & -7.4 & 1.70 & 0.114 & 0.124 \\
4.3 & 2 & 0.16 & 0.39 & 0.78 & -872 & 25 & -7.5 & 1.53 & 0.097 & 0.109 \\
4.4 & 2 & 0.16 & 0.78 & 0 & -131 & 7.8 & -1.30 & 1.32 & 0.199 & 0.199 \\
\hline 4.5 & 1.1 & 0.19 & 0.94 & 0 & -98 & 9 & -1.3 & 2.25 & 0.122 & 0.158 \\
4.6 & 1.1 & 0.19 & 0.47 & 0 & -1304 & 20 & -7.7 & 2.42 & 0.122 & 0.141 \\
\hline 4.7 & 0.4 & 0.08 & 0.40 & 0 & -1563 & 34 & -4.6 & 4.37 & 0.041 & 0.042 \\
4.8 & 0.4 & 0.08 & 0.40 & 0.5 & -1848 & 33 & -5.5 & 4.59 & 0.028 & 0.031 \\
\hline 4.9 & 0.24 & 0.08 & 0.20 & 0 & -1094 & 233 & -0.47 & 12.6 & 0.007 & 0.011 \\
4.10 & 0.24 & 0.08 & 0.40 & 0 & -1250 & 38 & -3.3 & 12.7 & 0.011 & 0.012 \\
4.11 & 0.24 & 0.08 & 0.40 & 0.5 & -1636 & 35 & -4.7 & 12.8 & 0.0099 & 0.011 \\
4.12 & 0.24 & 0.08 & 0.40 & 1 & -1649 & 34 & -5.0 & 12.7 & 0.0061 & 0.0068 \\
\hline \hline 4.13 & 1.28 & 0.24 & 0.17 & 0 & -399 & 229 & -1.6 & 19.1 & 0.097 & 0.100 \\
4.14 & 2.56 & 0.42 & 0.28 & 0.8 & 12724 & 257 & 8.5 & 16.8 & 0.120 \\
4.15 & 2.56 & 0.42 & 0.4 & 0.4 & -482 & 9.2 & -9.0 & 16.2 & 0.112 \\
\hline 4.16 & 2.56 & 0.61 & 0.42 & 1.21 & -970 & 10 & -5.4 & 36.5 & 0.030 & 0.04 \\
4.17 & 2.56 & 0.61 & 0.42 & 0.4 & -885 & 10 & -5.0 & 33.3 & 0.048 & 0.067 \\
\hline
\end{tabular}

Table 4. Voltages on the d.c. electrodes required to produce the results presented in table 3

\begin{tabular}{|l|l|l|l|}
\hline Result & $V_{1}$ & $V_{2}$ & $V_{3}$ \\
\hline units & $(\mathrm{V})$ & $(\mathrm{V})$ & $(\mathrm{V})$ \\
\hline 4.1 & -1.8451 & 1.4055 & -3.2924 \\
4.2 & -1.6997 & 2.2963 & -3.1155 \\
4.3 & -1.8592 & 2.4805 & -3.3316 \\
4.4 & -0.0885 & 0.3648 & -1.6934 \\
\hline 4.5 & -0.0290 & 0.2095 & -1.6090 \\
4.6 & -0.7951 & 1.4055 & -2.5750 \\
\hline 4.7 & -1.4109 & 2.0817 & -3.1413 \\
4.8 & -1.4960 & 2.1987 & -3.0247 \\
\hline 4.9 & -11.582 & 10.442 & -6.3685 \\
4.10 & -1.3316 & 1.9730 & -3.0171 \\
4.11 & -1.2679 & 1.8959 & -2.7263 \\
4.12 & -1.1612 & 1.9083 & -2.5576 \\
\hline \hline 4.13 & -22.030 & 4.7513 & -4.5768 \\
4.14 & 25.107 & -5.2939 & 18.252 \\
4.15 & -0.3793 & 0.1728 & -1.3831 \\
\hline 4.16 & -0.1479 & 0.1103 & -0.8449 \\
4.17 & -0.1427 & 0.0917 & -0.7954 \\
\hline
\end{tabular}

Table 5. The two cases studied which produced almost-octupoles above the plane of the electrodes. Result 5.1 uses the single plane electrode geometry shown in figure 17 Result 5.2 uses the "railway track" geometry shown in figure 18

\begin{tabular}{|l|l|l|l|l||l|l|l|l||l|l|}
\hline Result & $V_{A}$ & $V_{B}$ & $V_{C}$ & $V_{E}$ & $10^{4} \beta$ & $E_{\max }$ & $\rho$ & $\gamma$ & $\mu_{x}$ & $\mu_{y}$ \\
\hline units & $(\mathrm{V})$ & $(\mathrm{V})$ & $(\mathrm{V})$ & $(\mathrm{mV})$ & $\left(\mathrm{V} / a^{4}\right)$ & $(\mathrm{V} / a)$ & $(a)$ & $\left(10^{-3}\right)$ & $\left(10^{-3}\right)$ & $\left(10^{-3}\right)$ \\
\hline 5.1 & -4.257 & 4.560 & -3.527 & -2.197 & -0.0155 & 44.74 & 0.829 & 0.198 & 10.4 & 10.5 \\
5.2 & 1.089 & 1.212 & -4.526 & -201 & 0.000247 & 20.47 & 2.5 & 0.189 & 3.5 & 3.9 \\
\hline
\end{tabular}


Table 6. Example of the effect of manufacturing imprecision. The first three rows show the voltages required for $\partial^{2} V / \partial x^{2}=\partial^{2} V / \partial y^{2}=\partial^{2} V / \partial z^{2}=0$ at the centre of the trap. $E_{z}(z=0)$ is the axial electric field at $z=0$ when this condition is fulfilled. $z_{c}$ is the displacement of the centre of mass of an ion pair in a $(1 / 2) m \omega_{1}^{2} z^{2}$ potential well in the presence of electric field $E_{z} \cdot V_{2}^{\text {null }}$ is the voltage on the displaced electrode which is required to null the electric field. In all cases the r.f. electrode voltage amplitude is $\sim 240 \mathrm{~V}$, so that $\omega_{r} \simeq 2 \omega_{1}$.

\begin{tabular}{|l|l|l|l||l|c|l|}
\hline direction & $V_{1}$ & $V_{2}$ & $V_{3}$ & $E_{z}(z=0)$ & $z_{c}=q E_{z} / m \omega_{1}^{2}$ & $V_{2}^{\text {null }}$ \\
\hline & $(\mathrm{V})$ & $(\mathrm{V})$ & $(\mathrm{V})$ & $\left(\mathrm{V} \mu \mathrm{m}^{-1}\right)$ & $(\mu \mathrm{m})$ & $(\mathrm{V})$ \\
\hline$x$ & 281.7 & 97.6 & 593.0 & -0.17 & 21 & 85.8 \\
$y$ & 281.6 & 100.7 & 593.0 & -0.058 & 7.3 & 97.4 \\
$z$ & 277.3 & 100.0 & 593.0 & -0.073 & 9.2 & 95.1 \\
\hline
\end{tabular}

